\title{
GAUGED GROMOV-WITTEN THEORY FOR SMALL SPHERES
}

\author{
EDUARDO GONZALEZ AND CHRIS WOODWARD
}

\begin{abstract}
We relate the genus zero gauged Gromov-Witten invariants of a smooth projective variety for sufficiently small area with equivariant GromovWitten invariants. As an application we deduce a gauged version of abelianization for Gromov-Witten invariants in the small area chamber. In the symplectic setting, we prove that any sequence of genus zero symplectic vortices with vanishing area has a subsequence that converges after gauge transformation to a holomorphic map with zero average moment map.
\end{abstract}

\section{INTRODUCTION}

Gauged Gromov-Witten invariants are generalizations of usual Gromov-Witten invariants to the case of symplectic manifolds with group action. These invariants are defined as integrals over moduli spaces of gauged versions of holomorphic maps, known in the symplectic approach as symplectic vortices, and depend on a choice of area form on the domain curve. In this paper we study the limit of the gauged Gromov-Witten invariants in the case of genus zero curves with area tending to zero.

Ideally one would like to define gauged Gromov-Witten invariants for arbitrary Hamiltonian group actions. Let $K$ be a compact Lie group, $X$ a Hamiltonian $K$ manifold equipped with a moment map $\Phi: X \rightarrow \mathfrak{k}^{\vee}$, an invariant compatible almost complex structure $J$, and let $\Sigma$ be a compact smooth complex curve. A gauged holomorphic map from $\Sigma$ to $X$ is a pair $(A, u)$ consisting of a connection $A$ on a principal $K$-bundle $P \rightarrow \Sigma$ together with a section $u$ of the associated fiber bundle $P(X):=P \times_{K} X$ that is holomorphic with respect to the complex structure determined by $J$ and $A$. The space of gauged holomorphic maps with bundle $P$ has a formal Hamiltonian action of the group of gauge transformations $\mathcal{K}(P)$. The moment map depends on a choice of area form $\operatorname{Vol}_{\Sigma} \in \Omega^{2}(\Sigma)$ and an invariant inner product $():, \mathfrak{k} \times \mathfrak{k} \rightarrow \mathbb{R}$ on the Lie algebra $\mathfrak{k}$ inducing an identification $\mathfrak{k} \rightarrow \mathfrak{k}^{\vee}$. The symplectic quotient of the space of gauged holomorphic maps by the group of gauge transformations is the moduli space of symplectic vortices [6], [12], [5], [29]

$$
M^{K}(P, X):=\left\{(A, u) \mid F_{A}+u^{*} P(\Phi) \operatorname{Vol}_{\Sigma}=0\right\} / \mathcal{K}(P),
$$

where $F_{A} \in \Omega^{2}(\Sigma, P(\mathfrak{k}))$ is the curvature of $A$ and $P(\Phi): P(X) \rightarrow P\left(\mathfrak{k}^{\vee}\right) \cong P(\mathfrak{k})$ the map induced by $\Phi$. Let $M^{K}(\Sigma, X)$ be the union of $M^{K}(P, X)$ over topological types

Partially supported by NSF grants DMS1104670, DMS0904358 and the Simons Center for Geometry and Physics. To appear in Math. Zeit. Final version available at www.springerlink.com. 
of bundles $P \rightarrow \Sigma$, and $M^{K}(\Sigma, X, d)$ the subspace of homology class $d \in H_{2}^{K}(X, \mathbb{Z})$. In the case that $X$ is compact, one obtains a natural compactification $\bar{M}^{K}(\Sigma, X, d)$ by allowing bubbling of $u$ in the fibers of $P(X)$, so that $u$ is a stable map to $P(X)$ as in Mundet [29] and Ott [31]; we call such a nodal vortex $(A, u)$ polystable and stable if it has finite automorphism group. Gauged Gromov-Witten invariants should be defined by virtual integration over the moduli space $\bar{M}^{K}(\Sigma, X, d)$.

The necessary virtual fundamental cycles are more easily defined for algebraic targets. Let $G$ be the complexification of $K$ and suppose that $X$ is a smooth projectively-embedded $G$-variety. If $P \rightarrow \Sigma$ is a principal $K$-bundle then any connection $A$ on $P$ defines a holomorphic structure on the $G$-bundle $P(G)=P \times_{K} G$ associated to $P$. If $u$ is a holomorphic section of the associated $X$ bundle $P(X)=$ $P(G) \times{ }_{G} X$ then the pair $(P(G), u)$ is by definition a morphism from $\Sigma$ to the quotient stack $X / G$. By Mundet's correspondence, [28], an irreducible pair $(A, u)$ is complexgauge-equivalent to a vortex iff the corresponding holomorphic pair $(P(G), u)$ satisfies a semistability condition generalizing Mumford-Seshadri semistability for vector bundles on curves. As before, allowing bubbling in the fibers gives rise to a moduli stack $\overline{\mathcal{M}}_{n}^{G}(\Sigma, X)$, compact once the homology class is fixed. If semistable gauged maps have finite stabilizer then $\overline{\mathcal{M}}_{n}^{G}(\Sigma, X)$ is a Deligne-Mumford stack whose underlying coarse moduli space is homeomorphic to $\bar{M}_{n}^{K}(\Sigma, X)$. The machinery of Behrend-Fantechi 2 yields virtual fundamental classes and the desired invariants.

The choice of area form gives rise a one-parameter family of Mundet semistability conditions. In order to study the dependence of the gauged Gromov-Witten invariants on this choice we introduce a real parameter $\rho$ and say that a $\rho$-vortex is a pair $(A, u)$ satisfying the vortex equations with area form $\rho \operatorname{Vol}_{\Sigma}$, or $\rho$-semistable if it is Mundet semistable with this choice. Let $\bar{M}_{n}\left(\mathbb{P}^{1}\right):=\bar{M}_{0, n}\left(\mathbb{P}^{1},\left[\mathbb{P}^{1}\right]\right)$ be the Fulton-MacPherson moduli space of stable maps to $\mathbb{P}^{1}$ of homology class $\left[\mathbb{P}^{1}\right]$. The gauged Gromov-Witten invariants

$$
\langle,\rangle_{d, \rho}: H_{G}(X, \mathbb{Q})^{n} \otimes H\left(\bar{M}_{n}\left(\mathbb{P}^{1}\right)\right) \rightarrow \mathbb{Q}
$$

for $n \in \mathbb{Z}_{\geq 0}, d \in H_{2}(X, \mathbb{Z})$ are defined by virtual integration. The appearance of the Fulton-MacPherson, rather than the usual Grothendieck-Knudsen moduli space, is a manifestation of the dependence of the moduli space on the choice of area form. As the stability parameter $\rho$ varies the gauged Gromov-Witten invariants are related by a wall-crossing formula [14. There are two interesting limits in which the area of the domain tends to infinity or zero. The former has been studied by GaioSalamon [12]. In certain cases, they show that the limit of any sequence of solutions is a holomorphic map to the symplectic quotient $X / / K$ and so obtain a relationship between the equivariant cohomology of $X$ and the quantum cohomology of $X / / K$.

The purpose of this paper is to study the opposite limit in which the area of the domain tends to zero. The space of holomorphic maps $\operatorname{Hom}\left(\mathbb{P}^{1}, X\right)$ from $\mathbb{P}^{1}$ to $X$ has a natural $K$-action given by composition with the action, and a formal symplectic structure given by integrating the pull-back of the symplectic form on $X$. A formal 
moment map is

$$
\phi: \operatorname{Hom}\left(\mathbb{P}^{1}, X\right) \rightarrow \mathfrak{k}^{\vee}, \quad u \mapsto \int_{\mathbb{P}^{1}} u^{*} \Phi \operatorname{Vol}_{\mathbb{P}^{1}}
$$

The map $\phi$ extends to the moduli space of parametrized stable maps $\bar{M}_{0, n}\left(\mathbb{P}^{1} \times\right.$ $X,(1, d))$, that is the moduli of $n$-marked stable maps of genus 0 with degree $(1, d)$ into $\mathbb{P}^{1} \times X$ where $1=\left[\mathbb{P}^{1}\right]$ is the unit in $H_{2}\left(\mathbb{P}^{1}\right)$. We say that a polystable zero-area vortex of homology class $d \in H_{2}(X, \mathbb{Z})$ is a stable map $u: C \rightarrow \mathbb{P}^{1} \times X$ from a genus zero nodal curve $C$ of class $(1, d)$ with $\phi(u)=0$. The first main result says that these are exactly the stable maps that appear in the small area limit:

Theorem 1.1. Suppose that $X$ is a compact Hamiltonian $K$-manifold, $\rho_{\nu} \rightarrow 0$ is a sequence of positive real numbers, and $\left(A_{\nu}, u_{\nu}\right)$ is a sequence of polystable $\rho_{\nu}$-vortices on $\mathbb{P}^{1}$ with target $X$ of fixed homology class and bundle $P$. Then $P$ is trivializable and there exists a sequence $k_{\nu} \in \mathcal{K}(P)$ of gauge transformations such that after passing to a subsequence, $k_{\nu} A_{\nu}$ converges in $C^{0}$ to the trivial connection on $P$ and $k_{\nu} u_{\nu}$ Gromov converges to a polystable zero-area vortex $u_{0}$. Conversely, any regular stable zero-area vortex is a limit of a sequence $\left(A_{\nu}, u_{\nu}\right)$ of $\rho_{\nu}$-vortices with $\rho_{\nu} \rightarrow 0$.

The fact that the limit is a holomorphic map should not be surprising: given a sequence of vortices with vanishing area, the curvature of the connection goes to zero. Therefore, if the sequence of connections converges then it must converge to a flat connection, which in genus zero is trivializable. From the symplectic point of view, the subtle part of the theorem is the statement that the limiting map has zero average moment map: this follows from a study of a sub-leading term in the vortex equations. A similar theorem for the case that $X$ is aspherical and $K$ is abelian is proved in the thesis of Jan Wehrheim [39].

The moduli space of $n$-marked polystable zero-area vortices is denoted $\bar{M}_{n}^{K}\left(\mathbb{P}^{1}, X\right)_{0}$. If $X$ is a smooth projectively-embedded $G$-variety then $\bar{M}_{n}^{K}\left(\mathbb{P}^{1}, X\right)_{0}$ is the coarse moduli space of a moduli stack $\overline{\mathcal{M}}_{n}^{G}\left(\mathbb{P}^{1}, X\right)_{0}$. Integration gives rise to zero-area gauged Gromov-Witten invariants $\langle,\rangle_{d, 0}$. The zero-area gauged Gromov-Witten invariants may be viewed as the invariant part of Givental's equivariant GromovWitten invariants [13], and standard techniques [18], 41] allow, in principle, their computation from Givental's invariants, as in Theorem 5.5 below. The second main result is a description of the gauged Gromov-Witten invariants in the small-area limit:

Theorem 1.2. Suppose that $X$ is a smooth projectively-embedded variety and $d \in$ $H_{2}^{G}(X, \mathbb{Z})$, and every 0 -semistable gauged map has finite automorphism group. There exists a $\rho_{0}>0$ such that for all $\rho<\rho_{0}$, there is an equivalence $\overline{\mathcal{M}}_{n}^{G}\left(\mathbb{P}^{1}, X, d\right)_{0} \rightarrow$ $\overline{\mathcal{M}}_{n}^{G}\left(\mathbb{P}^{1}, X, d\right)_{\rho}$ of Deligne-Mumford stacks with relative perfect obstruction theories and an equality of invariants $\langle\alpha ; \beta\rangle_{d, \rho}=\langle\alpha ; \beta\rangle_{d, 0}$ for all $\alpha \in H_{G}(X, \mathbb{Q})^{n}$ and $\beta \in$ $H\left(\bar{M}_{n}\left(\mathbb{P}^{1}\right), \mathbb{Q}\right)$. 
Using this result we obtain a version of the abelianization conjecture of Bertram, Ciocan-Fontanine, and Kim [4] in the "small area chamber" relating the GromovWitten invariants for $G$ and a maximal torus $T$, in the case that $G$ is connected. Let $W$ be the Weyl group of $T \subset G$.

Theorem 1.3. Let $X$ be a smooth projectively-embedded $G$-variety such that every 0 -stable gauged map has finite automorphism group and $d_{G} \in H_{2}^{G}(X, \mathbb{Z})$. There exists a constant $\rho_{0}>0$ such that if $\rho<\rho_{0}$ and $\alpha \in H_{G}(X, \mathbb{Q})^{n}$ is an equivariant Hodge class (that is, a Chern character of an equivariant algebraic vector bundle) and $\beta \in H\left(\bar{M}_{n}\left(\mathbb{P}^{1}\right), \mathbb{Q}\right)$ then

$$
\langle\alpha, \beta\rangle_{G, d_{G}, \rho}=(\# W)^{-1} \sum_{d_{T} \mapsto d_{G}}\langle\alpha, \beta\rangle_{T, d_{T}, \rho}^{t w i s t}
$$

where the right hand side is a sum of gauged Gromov-Witten invariants twisted by the bundle with fiber $\mathfrak{g} / \mathfrak{t}$, where $\mathfrak{g}$ is the Lie algebra of $G$ and $\mathfrak{t} \subset \mathfrak{g}$ a Cartan subalgebra. All of these gauged Gromov-Witten invariants vanish unless $d_{G}$ is in the image of $H_{2}(X)$ in $H_{2}^{G}(X)$.

Combining this with the wall-crossing formula of [14 gives an abelianization formula for all chambers, in particular, the large area chamber which is related to the Gromov-Witten theory for the symplectic quotient.

A word on notation: we generally use the notation $M$ for a coarse moduli space (space of isomorphism classes), $\mathcal{M}$ for a Deligne-Mumford stack, and $\mathfrak{M}$ for an Artin stack, so the "ornateness" of the notation corresponds to "stackiness".

\section{Symplectic vortices and Gauged Gromov-Witten invariants}

In this section we recall the moduli spaces of symplectic vortices associated to Hamiltonian $K$-manifolds, introduced by Mundet [29] and Salamon and collaborators [5], 6], and the associated gauged Gromov-Witten invariants introduced (in the case of algebraic target) in [14.

2.1. Symplectic vortices. Let $\Sigma$ be a compact smooth complex curve with complex structure $J_{\Sigma}: T \Sigma \rightarrow T \Sigma$, and $\pi: P \rightarrow \Sigma$ a smooth principal $K$-bundle. Let $X$ be a compact Hamiltonian $K$-manifold with symplectic form $\omega$ and moment map $\Phi: X \rightarrow \mathfrak{k}^{\vee}$. The action of $K$ on $X$ gives rise to a homomorphism of Lie algebras

$$
\mathfrak{k} \rightarrow \operatorname{Vect}(X), \quad \xi \mapsto \xi_{X}, \quad \xi_{X}(x)=\left.\frac{d}{d t}\right|_{t=0} \exp (-t \xi) x .
$$

Our sign convention for the moment map is $\iota\left(\xi_{X}\right) \omega=-\mathrm{d}\langle\Phi, \xi\rangle, \forall \xi \in \mathfrak{k}$. By equivariant formality of Hamiltonian $K$-manifolds (see e.g. [17]) the second equivariant homology splits $H_{2}^{K}(X, \mathbb{Q}) \cong H_{2}(X, \mathbb{Q}) \oplus H_{2}^{K}(\mathrm{pt}, \mathbb{Q})$. If the action of $K$ extends to the complexification $G$ then we identify $H^{K}(X)$ and $H^{G}(X)$; generally speaking we use $H^{G}(X)$ when $X$ is a $G$-manifold, or $H^{K}(X)$ when we are using only the $K$-action. Continuous sections $u$ of $P(X):=(P \times X) / K$ correspond to lifts 
$u_{K}: \Sigma \rightarrow X_{K}=E K \times_{K} X$ of a classifying map $\Sigma \rightarrow B K$ for $P$ to $X_{K}$. Denote by $\pi_{P(X)}$ the projection of $P(X)$ onto $\Sigma$. The equivariant homology class $\mu(u) \in H_{2}^{K}(X, \mathbb{Z})$ of $u: \Sigma \rightarrow P(X)$ is defined by $\mu(u)=u_{K, *}[\Sigma]$. Let $\mathcal{J}(X)$ denote the space of compatible almost complex structures on $X$. The action of $K$ induces an action on $\mathcal{J}(X)$ by conjugation, and we denote by $\mathcal{J}(X)^{K}$ the invariant subspace. Let $\mathcal{A}(P)$ denote the space of smooth connections on $P$, and by $P(\mathfrak{k}):=(P \times \mathfrak{k}) / K$ the adjoint bundle. The space $\mathcal{A}(P)$ is an affine space modelled on $\Omega^{1}(\Sigma, P(\mathfrak{k}))$, with action given by $A \mapsto A+\pi^{*} a$ where $\pi^{*}: \Omega^{1}(\Sigma, P(\mathfrak{k})) \rightarrow \Omega^{1}(P, \mathfrak{k})^{K}$ is the pull-back map. For any $A \in \mathcal{A}(P)$, we denote $F_{A} \in \Omega^{2}(\Sigma, P(\mathfrak{k}))$ the curvature of $A$. Any connection $A \in \mathcal{A}(P)$ induces a map of spaces of almost complex structures

$$
\mathcal{J}(X)^{K} \rightarrow \mathcal{J}(P(X)), \quad J \mapsto J_{A}
$$

using the splitting defined by the connection. Let $\Gamma(\Sigma, P(X))$ denote the space of smooth sections of $P(X)$. Let

$$
\bar{\partial}_{A}: \Gamma(\Sigma, P(X)) \rightarrow \Omega^{0,1}\left(\Sigma,(\cdot)^{*} T^{\mathrm{vert}} P(X)\right), \quad \bar{\partial}_{A} u=\frac{1}{2}\left(\mathrm{~d} u+J_{A}(u) \circ \mathrm{d} u \circ J_{\Sigma}\right)
$$

be the Cauchy-Riemann operator defined by $J_{A}$. A gauged map to $X$ is a pair $(A, u) \in \mathcal{A}(P) \times \Gamma(\Sigma, P(X))$. Suppose $\operatorname{Vol}_{\Sigma}$ is the area form determined by a choice of metric on $\Sigma$. The energy of a gauged section $(A, u)$ is given by

$$
E(A, u)=\frac{1}{2} \int_{\Sigma}\left(\left|\mathrm{d}_{A} u\right|^{2}+\left|F_{A}\right|^{2}+\left|u^{*} P(\Phi)\right|^{2}\right) \operatorname{Vol}_{\Sigma} .
$$

The equivariant area $D(u)$ of $u$ is pairing of the class $\mu(u)$ with the class $\left[\omega_{K}\right] \in$ $H_{K}^{2}(X)$ of the equivariant symplectic form. The energy and equivariant area are related by [5, 3.1]

$$
E(A, u)=D(u)+\int_{\Sigma}\left(\left|\bar{\partial}_{A} u\right|^{2}+\frac{1}{2}\left|F_{A}+u^{*} P(\Phi) \operatorname{Vol}_{\Sigma}\right|^{2}\right) \operatorname{Vol}_{\Sigma} .
$$

The space of gauged holomorphic maps with underlying bundle $P$ is

$$
\mathcal{H}(P, X)=\left\{(A, u) \in \mathcal{A}(P) \times \Gamma(\Sigma, P(X)), \quad \bar{\partial}_{A} u=0\right\} .
$$

The space $\mathcal{H}(P, X)$ has a formal symplectic form induced from the sum of the formal symplectic forms on the factors, given as follows. Let

$$
\Omega^{1}(P(\mathfrak{k}))^{2} \rightarrow \mathbb{R}, \quad\left(a_{1}, a_{2}\right) \mapsto \int_{\Sigma}\left(a_{1} \wedge a_{2}\right)
$$

denote the symplectic form on the affine space of connections $\mathcal{A}(P)$ determined by the metric on $\mathfrak{k}$. On the other hand, let $P(\omega)$ denote the fiber-wise two-form on $P(X)$ defined by $\omega$. Choose a two-form $\operatorname{Vol}_{\Sigma} \in \Omega^{2}(\Sigma)$ and define

$$
\Omega^{0}\left(\Sigma, u^{*} T^{\mathrm{vert}} P(X)\right)^{2} \rightarrow \mathbb{R}, \quad\left(v_{1}, v_{2}\right) \mapsto \int_{\Sigma}\left(u^{*} P(\omega)\right)\left(v_{1}, v_{2}\right) \mathrm{Vol}_{\Sigma}
$$

Choose a constant $\rho>0$, called the vortex parameter, and consider the formal two-form

$$
\left(\left(a_{1}, v_{1}\right),\left(a_{2}, v_{2}\right)\right) \rightarrow \int_{\Sigma}\left(a_{1} \wedge a_{2}\right)+\rho \operatorname{Vol}_{\Sigma}\left(u^{*} P(\omega)\right)\left(v_{1}, v_{2}\right)
$$


Let $\mathcal{K}(P)$ denote the group of gauge transformations. The Lie algebra $\mathfrak{k}(P)$ of $\mathcal{K}(P)$ is the space of sections $\Omega^{0}(\Sigma, P(\mathfrak{k}))$ of the adjoint bundle. The action of $\mathcal{K}(P)$ on the space of pairs generates an infinitesimal action $(A, u) \mapsto\left(\mathrm{d}_{A} \xi, u^{*} \xi_{X}\right)$ where $\xi_{X} \in \Omega^{0}\left(\Sigma, P \times_{K} \operatorname{Vect}(X)\right)$ is the vertical vector field induced by $\xi$. The action preserves the formal two-form (4) and has moment map given by the curvature plus pull-back of the moment map for $X$,

$$
\mathcal{A}(P) \times \Gamma(\Sigma, P(X)) \rightarrow \Omega^{2}(\Sigma, P(\mathfrak{k})), \quad(A, u) \mapsto F_{A, u}:=F_{A}+\rho \operatorname{Vol}_{\Sigma} u^{*} P(\Phi) .
$$

By restriction one obtains a formal Hamiltonian action on $\mathcal{H}(P, X)$. These formal considerations motivate the following definition.

Definition 2.1. A gauged holomorphic map $(A, u)$ is a $\rho$-vortex iff $F_{A, u}=0$.

The moduli space of $\rho$-vortices of class $d \in H_{2}^{K}(X)$ with bundle $P$ is

$$
M^{K}(P, X, d)_{\rho}:=\left\{F_{A, u}=0, \mu(u)=d\right\} / \mathcal{K}(P) .
$$

Let $M^{K}(P, X)_{\rho}$ be the union over classes $d$, and $M^{K}(\Sigma, X)_{\rho}$ the union over types $P$. Note that the first Chern class of $P$ is determined by the homology class of $u_{K}$ via the projection $X_{K} \rightarrow B K$. The formal tangent space to $M^{K}(P, X)_{\rho}$ is the kernel of a Fredholm operator given as follows. We first give the spaces of connections and sections the structure of Banach manifolds by taking completions with respect to Sobolev norms $\|\cdot\|_{k, p}$ for positive integers $k, p$. For $p>2$, define

$$
\begin{aligned}
& \mathrm{d}_{A, u, \rho}: \Omega^{1}(\Sigma, P(\mathfrak{k}))_{1, p} \oplus \Omega^{0}\left(\Sigma, u^{*} T^{\mathrm{vert}} P(X)\right)_{1, p} \rightarrow \Omega^{2}(\Sigma, P(\mathfrak{k}))_{0, p} \\
&(a, v) \mapsto \mathrm{d}_{A} a+\rho \operatorname{Vol}_{\Sigma} L_{v} P(\Phi) \\
& \mathrm{d}_{A, u, \rho}^{*}: \Omega^{1}(\Sigma, P(\mathfrak{k}))_{1, p} \oplus \Omega^{0}\left(\Sigma, u^{*} T^{\mathrm{vert}} P(X)\right)_{1, p} \rightarrow \Omega^{0}(\Sigma, P(\mathfrak{k}))_{0, p} \\
&(a, v) \mapsto \mathrm{d}_{A}^{*} a+\rho \operatorname{Vol}_{\Sigma} u^{*} L_{J v} P(\Phi) .
\end{aligned}
$$

Here $L_{v} P(\Phi) \in \Omega^{0}(\Sigma, P(\mathfrak{k}))$ denotes the derivative of $P(\Phi)$ in the direction of $v$. The set

$$
S_{A, u}=\left\{\left(A+\pi^{*} a, \exp _{u}(v)\right),(a, v) \in \operatorname{kerd}_{A, u, \rho}^{*}\right\} \subset \mathcal{H}(P, X)_{1, p}
$$

contains a slice for the action of $\mathcal{K}(P)_{2, p}$ on $\mathcal{H}(P, X)_{1, p}$ at $(A, u)$ (see [5, Section 4]). Define

$$
\begin{aligned}
& \mathcal{F}_{A, u}^{\rho}: \Omega^{1}(\Sigma, P(\mathfrak{k}))_{1, p} \oplus \Omega^{0}\left(\Sigma, u^{*} T^{\mathrm{vert}} P(X)\right)_{1, p} \\
& \rightarrow\left(\Omega^{0} \oplus \Omega^{2}\right)(\Sigma, P(\mathfrak{k}))_{0, p} \oplus \Omega^{0,1}\left(\Sigma, u^{*} T^{\mathrm{vert}} P(X)\right)_{0, p} \\
&(a, v) \mapsto\left(F_{A+\pi^{*} a}+\rho \operatorname{Vol}_{\Sigma} \exp _{u}(v)^{*} \Phi, \mathrm{d}_{A, \rho}^{*}(a, v), \Psi_{u}(v)^{-1} \bar{\partial}_{A+\pi^{*} a} \exp _{u}(v)\right)
\end{aligned}
$$

where $\Psi_{u}(v)^{-1}$ is parallel transport from $\exp _{u}(v)$ to $u$ using the Hermitian modification of the Levi-Civita connection [27, Section 3.1]. Let $D_{A, u}$ denote the linearization of the Cauchy-Riemann operator for $J_{A}$,

$$
D_{A, u}(a, v):=\left(\nabla_{A} v\right)^{0,1}+a_{X}^{0,1}+\frac{1}{2} J_{u}\left(\nabla_{A, v} J\right)_{u} \partial_{A} u
$$


where $a_{X}$ denotes the image of $a$ under the map $\Omega^{1}(\Sigma, P(\mathfrak{k})) \rightarrow \Omega^{1}\left(\Sigma, u^{*} T^{\text {vert }} P(X)\right)$ induced by the action, and 0,1 denotes projection on the 0,1 -component. The last term vanishes if $J$ is integrable.

Definition 2.2. The linearized operator for $(A, u) \in \mathcal{H}(P, X)$ and vortex parameter $\rho$ is the operator

$$
\begin{aligned}
& \tilde{D}_{A, u}^{\rho}: \Omega^{1}(\Sigma, P(\mathfrak{k})) \oplus \Omega^{0}\left(\Sigma, u^{*} T^{\mathrm{vert}}(P(X))\right. \\
& \rightarrow\left(\Omega^{0} \oplus \Omega^{2}\right)(\Sigma, P(\mathfrak{k})) \oplus \Omega^{0,1}\left(\Sigma, u^{*} T^{\mathrm{vert}}(P(X))\right. \\
&(a, v) \mapsto\left(\mathrm{d}_{A, u, \rho}(a, v), \mathrm{d}_{A, u, \rho}^{*}(a, v), D_{A, u}(a, v)\right) .
\end{aligned}
$$

$(A, u)$ is regular if the operator $\tilde{D}_{A, u}^{\rho}$ is surjective. If $(A, u)$ is regular then it follows from the slice condition that $\operatorname{aut}(A, u)$ is trivial, and since $\operatorname{Aut}(A, u) \subset \operatorname{Aut}(A)$ is compact, $\operatorname{Aut}(A, u)$ is finite, that is, $(A, u)$ is stable. The space of infinitesimal deformations of $(A, u)$ is $\operatorname{Def}(A, u)=\operatorname{ker}\left(\tilde{D}_{A, u}^{\rho}\right)$.

The operator $\tilde{D}_{A, u}^{\rho}$ is the linearization of the map $\mathcal{F}_{A, u}^{\rho}$ at $(A, u)$. It is elliptic, and so has finite dimensional kernel and cokernel in the Sobolev completions above. The following theorem, due to Mundet, Salamon et al. [29, [5], generalizes the standard results for pseudoholomorphic maps to the gauged setting:

Theorem 2.3. For any constants $c_{1}, c_{2}>0$, the set of elements $[A, u] \in M^{K}(\Sigma, X)_{\rho}$ with $\sup \left|\mathrm{d}_{A} u\right|<c_{1}$ and $E(A, u)<c_{2}$ is compact. The regular locus $M^{K, \operatorname{reg}}(\Sigma, X)_{\rho}$ is a smooth orbifold with tangent space at $[A, u]$ isomorphic to $\operatorname{Def}(A, u)$. The dimension of the component of homology class $d \in H_{2}^{K}(X)$ is given by

$\operatorname{dim}\left(M^{K, \operatorname{reg}}(\Sigma, X, d)_{\rho}\right)=\operatorname{Ind}\left(\tilde{D}_{A, u}^{\rho}\right)=(1-g)(\operatorname{dim}(X)-2 \operatorname{dim}(K))+2\left(c_{1}^{K}(T X), d\right)$ where $g=\operatorname{genus}(\Sigma)$.

Spaces with markings and framings are given as follows.

Definition 2.4. An $n$-marked symplectic vortex is a vortex $(A, u)$ together with $n$-tuple $\underline{z}=\left(z_{1}, \ldots, z_{n}\right)$ of distinct points on $\Sigma$. An isomorphism of $n$-marked symplectic vortices is an isomorphism of the underlying vortices, such that the markings are equal. A framed vortex is a collection $(A, u, \underline{z}, \underline{\phi})$, where $(A, u, \underline{z})$ is a marked vortex and $\phi=\left(\phi_{1}, \ldots, \phi_{n}\right)$ are trivializations of the fibers of $P$ at $z_{1}, \ldots, z_{n}$, that is, each $\phi_{j}: P_{z_{j}}^{-} \rightarrow K$ is a $K$-equivariant isomorphism. An isomorphism of framed vortices is an isomorphism of the underlying marked vortices, intertwining the framings: if $\psi: P \rightarrow P^{\prime}$ denotes the bundle isomorphism, then $\phi_{j}=\phi_{j}^{\prime} \circ \psi, j=1, \ldots, n$.

Let $M_{n}^{K}(\Sigma, X)_{\rho}$ denote the moduli space of isomorphism classes $n$-marked $\rho$-vortices. The moduli space $M_{n}^{K}(\Sigma, X)_{\rho}$ is homeomorphic to the product $M^{K}(\Sigma, X)_{\rho} \times M_{n}(\Sigma)$ where $M_{n}(\Sigma)$ denotes the configuration space of $n$-tuples of distinct points on $\Sigma$. Let $M_{n}^{K, \mathrm{fr}}(\Sigma, X)_{\rho}$ denote the moduli space of isomorphism classes of framed $n$-marked $\rho$-vortices. The framed evaluation map is

$$
\mathrm{ev}^{\mathrm{fr}}: M_{n}^{K, \mathrm{fr}}(\Sigma, X)_{\rho} \rightarrow X^{n}, \quad[A, u, \underline{z}, \underline{\phi}] \mapsto\left(\phi_{1}\left(u\left(z_{1}\right)\right), \ldots, \phi_{n}\left(u\left(z_{n}\right)\right)\right)
$$


defined by combining the framings with evaluation at the marked points. Define

$$
\varphi: M_{n}^{K, \mathrm{fr}}(\Sigma, X)_{\rho} \rightarrow M_{n}^{K}(\Sigma, X)_{\rho}, \quad[A, u, \underline{z}, \underline{\phi}] \mapsto[A, u, \underline{z}]
$$

by forgetting the framings. Since the action of the gauge group admits slices (5) over the regular locus the map $M_{n}^{K \text {,fr,reg }}(\Sigma, X)_{\rho}$ the map $\varphi$ is an orbifold principal $K^{n}$-bundle. Suppose that $K^{n}$ acts freely, so that $M_{n}^{K \text {,fr,reg }}(\Sigma, X)_{\rho}$ is an honest bundle. Let $\psi: M_{n}^{K, \text { fr,reg }}(\Sigma, X)_{\rho} \rightarrow E K^{n}$ be a classifying map for $\varphi$. Combining $\psi$ with the framed evaluation map $\mathrm{ev}^{\mathrm{fr}}$ gives rise to a $K^{n}$-equivariant map $\mathrm{ev}^{\mathrm{fr}} \times \psi$ : $M_{n}^{K, \text { fr,reg }}(\Sigma, X)_{\rho} \rightarrow X^{n} \times E K^{n}$. Define the evaluation map $\mathrm{ev}_{\mathbb{Z}}: M_{n}^{K, \mathrm{reg}}(\Sigma, X)_{\rho} \rightarrow$ $\left(X^{n} \times E K^{n}\right) / K^{n}=X_{K}^{n}$ by descending $\mathrm{ev}^{\mathrm{fr}} \times \psi$ to the quotient. Pull-back by $\mathrm{ev}_{\mathbb{Z}}$ induces a map in equivariant cohomology with integral coefficients. More generally, the classifying map exists after passing to the classifying space of $M_{n}^{K \text {,fr,reg }}(\Sigma, X)_{\rho}$, and we obtain a pull-back for cohomology in rational coefficients

$$
\mathrm{ev}^{*}: H_{K}(X, \mathbb{Q})^{n} \rightarrow H\left(M_{n}^{K, \mathrm{reg}}(\Sigma, X)_{\rho}, \mathbb{Q}\right) .
$$

2.2. Compactification. There is a natural compactification of the moduli space of vortices obtained by allowing bubbling:

Definition 2.5. Let $\Sigma$ be a compact connected smooth complex curve and $X$ a Hamiltonian $K$-manifold. A nodal gauged holomorphic map to $X$ with principal component $\Sigma$ consists of a datum $(P, A, C, u, \underline{z})$ where $P$ is a principal $K$-bundle on $\Sigma ; A$ is a connection on $P$; $C$ is a compact nodal curve, $u: C \rightarrow P(X)$ is a holomorphic map such that the composition $\pi_{P(X)} \circ u: C \rightarrow \Sigma$ has homology class $[\Sigma] ; \underline{z}=\left(z_{1}, \ldots, z_{n}\right) \in C^{n}$ are distinct, smooth points of $C$. The principal component of a nodal gauged holomorphic map is the (unmarked) gauged holomorphic map ( $\left.P, A, C_{0}, u_{0}\right)$ where $C_{0} \subset C$ is the unique component of $C$ on which the composition $\pi_{P(X)} \circ u \mid C_{0}$ is non-constant. A nodal vortex is a nodal gauged map such that the principal component $\left(A, u_{0}\right)$ is a vortex. A nodal vortex is polystable if the underlying map $u$ is stable. A polystable vortex is stable if it has finite automorphism group. An isomorphism of nodal $\rho$-vortices $(P, A, C, u, \underline{z}),\left(P^{\prime}, A^{\prime}, C^{\prime}, u^{\prime}, \underline{z}^{\prime}\right)$ consists of an isomorphism of nodal curves $f: C \rightarrow C^{\prime}$ and a bundle isomorphism $k: P \rightarrow P^{\prime}$ such that $k A=A^{\prime}, u^{\prime} \circ f=k u$, and $f\left(z_{i}\right)=z_{i}^{\prime}, i=1, \ldots, n$. A framed nodal vortex consists of a nodal vortex together with framings at the attaching points of the bubbles $\phi_{i}: P_{\hat{z}_{i}} \rightarrow K, i=1, \ldots, n$. The combinatorial type of a gauged nodal map $(P, A, C, u, \underline{z})$ is the rooted graph $\Gamma$ whose vertices are the components of $C$, whose finite edges represent the nodes $w_{i}^{ \pm}, i=1, \ldots, m$, semi-infinite edges represent the markings $z_{1}, \ldots, z_{n}$, and whose root vertex represents the principal component $C_{0}$. The homology class of a nodal vortex is the class $u_{K, *}[C] \in H_{2}^{K}(X, \mathbb{Z})$ where $u_{K}: C \rightarrow E K \times_{K} X$ is the lift of a classifying map $C \rightarrow E K$ of $\left(\pi_{P(X)} \circ u\right)^{*} P \rightarrow C$ corresponding to $u$.

We often abbreviate the data of a nodal vortex as $(A, u)$ to save space. Let $M_{n, \Gamma}^{K}(\Sigma, X, d)_{\rho}$ resp. $M_{n, \Gamma}^{K, \text { fr }}(\Sigma, X, d)_{\rho}$ be the moduli space of isomorphism classes of 
polystable resp. framed polystable $\rho$-vortices of combinatorial type $\Gamma$ and homology class $d \in H_{2}^{K}(X, \mathbb{Z})$, and $\bar{M}_{n}^{K}(\Sigma, X, d)_{\rho}$ be the union over types

$$
\bar{M}_{n}^{K}(\Sigma, X, d)_{\rho}=\bigcup_{\Gamma} M_{n, \Gamma}^{K}(\Sigma, X, d)_{\rho} .
$$

A notion of Gromov convergence of nodal vortices, described in 31, defines a topology on $\bar{M}_{n}^{K}(\Sigma, X, d)_{\rho}$.

To define the linearized operator associated to a vortex, we recall that the normalization $\tilde{C}$ of a nodal curve $C$ is the disjoint union of the irreducible components. Let $\Omega^{0}\left(C, u^{*} T^{\text {vert }} P(X)\right)_{1, p}$ denote the subspace of $\Omega^{0}\left(\tilde{C}, u^{*} T^{\text {vert }} P(X)\right)_{1, p}$ consisting of sections that agree at the nodes.

Definition 2.6. Given a nodal vortex $(A \in \mathcal{A}(P), u: C \rightarrow P(X))$, let $\tilde{D}_{A, u}^{\rho}$ denote the linearized operator

$$
\begin{aligned}
& \Omega^{1}(\Sigma, P(\mathfrak{k}))_{1, p} \oplus \Omega^{0}\left(C, u^{*} T^{\mathrm{vert}} P(X)\right)_{1, p} \\
& \rightarrow\left(\Omega^{0} \oplus \Omega^{2}\right)(\Sigma, P(\mathfrak{k}))_{0, p} \oplus \Omega^{0,1}\left(\tilde{C}, u^{*} T^{\mathrm{vert}} P(X)\right)_{0, p} \\
& \quad(a, v) \mapsto\left(\tilde{D}_{A, u_{0}}\left(a, v_{0}\right),\left(D_{u_{j}} v_{j}\right)_{j=1}^{k}\right)
\end{aligned}
$$

given by the operator $\tilde{D}_{A, u}^{\rho}$ on the principal component and the linearized CauchyRiemann operator $\tilde{D}_{v_{j}}$ on the bubbles. We say that $(A, u)$ is regular iff $\tilde{D}_{A, u}^{\rho}$ is surjective.

Theorem 2.7. Let $X$ be a compact Hamiltonian $K$-manifold. For any $c>0$, the union of components $\bar{M}_{n}^{K}(\Sigma, X, d)_{\rho}$ with $\left\langle d,\left[\omega_{K}\right]\right\rangle<c$ is a compact, Hausdorff space. The regular locus $\bar{M}^{K, \text { reg }}(\Sigma, X, d)_{\rho}$ has the structure of a partially smooth topological (non-canonically $C^{1}$ ) orbifold.

This generalization of Gromov compactness is proved in Ott [31, with special cases proved previously in Mundet [29]. That the topology defined by Gromov convergence is compact follows using local distance functions as in [27, p. 134]. The orbifold charts are given by universal deformations constructed in [15]. The evaluation maps on the principal stratum defined in (8) extend to maps

$$
\mathrm{ev}^{*}: H_{K}(X, \mathbb{Q})^{n} \rightarrow H\left(\bar{M}_{n}^{K, \mathrm{reg}}(\Sigma, X)_{\rho}, \mathbb{Q}\right)
$$

and the forgetful morphism extends to map $f: \bar{M}_{n}^{K}(\Sigma, X)_{\rho} \rightarrow \bar{M}_{n}^{K}(\Sigma)$ forgetting $A$, replacing $u$ with its composition with the projection $\pi_{P(X)}$, and collapsing any unstable component.

2.3. Semistable gauged maps. Let $X$ be a smooth projectively-embedded $G$ variety. Recall that a principal $G$-bundle over a scheme $S$ is an $S$-scheme $P$ with a right $G$-action such that $P$ is locally trivial in the étale topology on $S$. 
Definition 2.8. An $n$-marked nodal gauged map to $X$ over a scheme $S$ with principal component $\Sigma$ consists of a datum $(P, C, u, \underline{z})$ where $(C, \underline{z})$ is a family of $n$-marked pre-stable curves (see e.g. Behrend-Manin [3]) of genus that of $\Sigma, P \rightarrow \Sigma \times S$ is a principal $G$-bundle; and $u: C \rightarrow P(X):=(P \times X) / G$ is a family of stable maps such that composition of $u$ with the projection $P(X) \rightarrow \Sigma$ has homology class $[\Sigma]$.

The restriction on the homology class and genus means that the domain $C$ of a nodal gauged map over a point $S=\{s\}$ has a principal component $C_{0}$ isomorphic to $\Sigma$ under the composition of $C \rightarrow P(X)$ with $P(X) \rightarrow \Sigma$ and a number of bubble components $C_{1}, \ldots, C_{m}$ mapping to points in $\Sigma$.

Mundet's stability condition [28, which combines that of Ramanathan 32] for principal bundles and Mumford, for finite-dimensional actions, is given as follows. Recall that a subgroup $R \subset G$ is parabolic iff $G / R$ is compact. A Levi subgroup of a parabolic subgroup $R$ is a maximal reductive subgroup. Each Levi subgroup has a maximal unipotent complementary subgroup $U \subset G$ such that $R=L U$. Quotienting by $U$ defines a surjective homomorphism $p: R \rightarrow L$. A parabolic reduction of a principal $G$-bundle $P$ consists of a parabolic subgroup $R \subset G$, a principal $R$-bundle $P^{\prime}$ and an isomorphism $P^{\prime}(G) \rightarrow P$. There is a canonical bijection between parabolic reductions with subgroup $R$ and sections $\sigma: \Sigma \rightarrow P / R$, given by $\sigma \mapsto \sigma^{*} P$, the pullback of the $R$-bundle $P \rightarrow P / R$ under $\sigma: \Sigma \rightarrow P / R$. Given such a section $\sigma$ let $p_{*} \sigma^{*} P$ denote the associated $L$-bundle. Let $i: L \rightarrow G$ be the inclusion.

Definition 2.9. Let $P \rightarrow \Sigma$ be a principal $G$-bundle and $u: \Sigma \rightarrow P(X)$ a section. Given a parabolic reduction $\sigma: \Sigma \rightarrow P / R$, the associated graded bundle is $\operatorname{Gr}(P)=$ $i_{*} p_{*} \sigma^{*} P$; note that $\operatorname{Gr}(P)$ has a canonical reduction to $L$ given by $p_{*} \sigma^{*} P$. Let $Z$ denote the center of $L, \mathfrak{z}$ its Lie algebra, and $\lambda \in \mathfrak{z}$ antidominant such that $\exp (\lambda)=1$. The family of automorphisms of $R$ given by conjugation by $z^{\lambda / 2 \pi i}=$ $\exp (\ln (z) \lambda / 2 \pi i)$ induces a family of bundles $P_{\sigma, \lambda}:=\left(\sigma^{*} P \times \mathbb{C}\right) \times_{R} R$ (the action of $R$ on itself is twisted by conjugation) with central fiber $\left(p_{*} \sigma^{*} P\right)(R)$. The stable section $u$ extends canonically over the generic fibers by multiplication by $z^{\lambda / 2 \pi i}$ and over the central fiber, by properness of stable maps, to an associated graded stable map denoted $\operatorname{Gr}(u)$ from a nodal curve $C$ to $(\operatorname{Gr}(P))(X)$. Suppose that $\lambda$ defines a weight of $L$ via the inner product on $\mathfrak{l} \subset \mathfrak{g}$. The degree of the pair $(\sigma, \lambda)$ is defined in terms of the associated graded by

$$
\mu_{\sigma, \lambda}(P, u)=\int_{\Sigma} c_{1}\left(p_{*} \sigma^{*} P \times_{L} \mathbb{C}_{\lambda}\right)+\rho \mu_{\lambda}\left(\operatorname{Gr}(u)_{0}\right) \operatorname{Vol}_{\Sigma}
$$

where $\operatorname{Gr}(u)_{0}: \Sigma \rightarrow P(X)$ denotes the principal component of $\operatorname{Gr}(u): C \rightarrow P(X)$, and $\mu_{\lambda}\left(\operatorname{Gr}(u)_{0}\right)$ is the weight of the one-parameter subgroup $\mathbb{C}_{\lambda}^{*}$ generated by $\lambda$ on any fiber $\mathcal{O}_{X}(1)_{\operatorname{Gr}(u)_{0}(z)}, z \in \Sigma$. The pair $(\sigma, \lambda)$ is destabilizing iff $\mu_{\sigma, \lambda}(P, u)>0$. $(P, u)$ is unstable if there exists a de-stabilizing pair $(\sigma, \lambda)$, semistable if it is not unstable, stable if there are no pairs with $\mu_{\sigma, \lambda}(P, u) \geq 0$, and polystable if it is semistable but not stable and $(P, u)$ is isomorphic to its associated graded for any pair $(\sigma, \lambda)$ satisfying the above with equality. 
The weight $\mu_{\lambda}\left(\operatorname{Gr}(u)_{0}\right)$ can be described in terms of the moment map as follows. The underlying smooth principal $G$-bundle admits a reduction of structure group from $G$ to $K$. Suppose that $X$ is equipped with moment map $\Phi: X \rightarrow \mathfrak{k}^{\vee} \cong \mathfrak{k}$ induced by the Fubini-Study equivariant symplectic form. The map $\Phi$ induces a map $P(\Phi): P(X) \rightarrow P(\mathfrak{k})$ taking values in $P(\mathfrak{l})$ on the $\lambda$-fixed locus. Being $L$ invariant $\lambda$ induces an element $P(\lambda) \in P(\mathfrak{l})$. Then

$$
\mu_{\lambda}\left(\operatorname{Gr}(u)_{0}\right)=\left\langle P(\Phi) \circ \operatorname{Gr}(u)_{0}(z), P(\lambda)\right\rangle, \forall z \in \Sigma .
$$

Let $\overline{\mathfrak{M}}_{n}^{G}(\Sigma, X)$ denote the category of nodal $n$-marked gauged maps to $X$ with principal component $\Sigma$.

Theorem 2.10. [14] $\overline{\mathfrak{M}}_{n}^{G}(\Sigma, X)$ has the structure of a (non-finite-type, non-separated) Artin stack. The subcategory $\overline{\mathcal{M}}_{n}^{G}(\Sigma, X)_{\rho}$ of $\rho$-semistable gauged maps has the structure of an open substack. If all automorphism groups are finite, $\overline{\mathcal{M}}_{n}^{G}(\Sigma, X)_{\rho}$ (or rather, any component $\overline{\mathcal{M}}_{n}^{G}(\Sigma, X, d)_{\rho}$ since $\overline{\mathcal{M}}_{n}^{G}(\Sigma, X)_{\rho}$ is not finite type) is a Deligne-Mumford stack equipped with a canonical perfect obstruction theory, and its coarse moduli space is homeomorphic to the moduli space of symplectic vortices $\bar{M}_{n}^{K}(\Sigma, X)_{\rho}$. For each constant $c>0$, the union of components $\overline{\mathcal{M}}_{n}^{G}(\Sigma, X, d)_{\rho}$ with homology class $d \in H_{2}^{G}(X)$ satisfying $\left\langle d,\left[\omega_{G}\right]\right\rangle<c$ is proper.

Let $\overline{\mathfrak{M}}_{n}(\Sigma)$ be the moduli stack for $X$ and $G$ trivial; this is the moduli stack of $n$-marked pre-stable maps to $\Sigma$ of class $[\Sigma]$ and genus that of $\Sigma$. A relative perfect obstruction theory for the morphism $\overline{\mathcal{M}}_{n}^{G}(\Sigma, X, d)_{\rho} \rightarrow \overline{\mathfrak{M}}_{n}(\Sigma)$ is defined by $R p_{*}\left(e^{*} T(X / G)\right)^{\vee}$, where $p: \overline{\mathcal{U}}_{n}^{G}(\Sigma, X, d)_{\rho} \rightarrow \overline{\mathcal{M}}_{n}^{G}(\Sigma, X, d)_{\rho}$ is the universal curve and $e: \overline{\mathcal{U}}_{n}^{G}(\Sigma, X, d)_{\rho} \rightarrow X / G$ the universal morphism, together with its canonical morphism to the cotangent complex. Let ev $: \overline{\mathcal{M}}_{n}^{G}(\Sigma, X)_{\rho} \rightarrow(X / G)^{n}$ denote the evaluation map at the marked points, $(P, C, u, \underline{z}) \mapsto\left(\underline{z}^{*} P, u \circ \underline{z}\right)$. Let ev* denote the induced pull-back in rational cohomology of coarse moduli spaces,

$$
\mathrm{ev}^{*}: H_{G}(X, \mathbb{Q})^{n} \rightarrow H\left(\bar{M}_{n}^{G}(\Sigma, X)_{\rho}, \mathbb{Q}\right) .
$$

As explained in [14] there exists a forgetful morphism $f: \overline{\mathcal{M}}_{n}^{G}(\Sigma, X)_{\rho} \rightarrow \overline{\mathcal{M}}_{n}(\Sigma):=$ $\overline{\mathcal{M}}_{\text {genus }(\Sigma), n}(\Sigma,[\Sigma])$ which maps $(C, P, u, \underline{z})$ to the stable map to $\Sigma$ obtained from $\left(C, \pi_{P(X)} \circ u, \underline{z}\right)$ by collapsing unstable components.

2.4. Gauged Gromov-Witten invariants. We assume that the reader is familiar with twisted Gromov-Witten invariants as in Coates-Givental [7. Given a $G$ equivariant vector bundle $V \rightarrow X$, define the index

$$
\operatorname{Ind}(V)=R p_{*} e^{*} V \in \operatorname{Ob}\left(D^{b} \operatorname{Coh}\left(\overline{\mathcal{M}}_{n}^{G}(\Sigma, X)_{\rho}\right)\right)
$$

where $p$, as before, is the projection from the universal curve and $e$ is the universal morphism. If $V$ is a $G$-representation, then by $\operatorname{Ind}(V)$ we mean the index of the 
trivial vector bundle with fiber $V$. Denote the Euler class of $\operatorname{Ind}(V)$ by

$$
\operatorname{Eul}_{\mathbb{C}^{*}}(\operatorname{Ind}(V)) \in H\left(\bar{M}_{n}^{G}(\Sigma, X)_{\rho}, \mathbb{Q}\right)\left[\zeta, \zeta^{-1}\right]
$$

here $\zeta$ the equivariant parameter for the action of $\mathbb{C}^{*}$ by scalar multiplication, acting trivially on the base.

Definition 2.11. Suppose that $X, G, \rho, d$ are as above so that every $\rho$-semistable gauged map of class $d$ has finite automorphism group. The $V$-twisted gauged GromovWitten invariant associated to $\alpha \in H_{G}(X)^{n}, \beta \in H\left(\bar{M}_{n}(\Sigma)\right)$ is

$$
\langle\alpha, \beta\rangle_{\rho, d, V}:=\int_{\left[\overline{\mathcal{M}}_{n}^{G}(\Sigma, X, d)_{\rho}\right]} \operatorname{ev}^{*} \alpha \cup f^{*} \beta \cup \operatorname{Eul}_{\mathbb{C}^{*}}(\operatorname{Ind}(V)) \in \mathbb{Q}\left[\zeta, \zeta^{-1}\right] .
$$

The splitting axiom for these invariants is discussed in [14]: they form a cohomological trace on the cohomological field theory defined by the equivariant GromovWitten invariants introduced by Givental [13. In the symplectic setting the invariants can be defined under the assumption that every vortex is regular. Pulling back and integrating using the usual orbifold fundamental class gives a symplectic definition of the gauged Gromov-Witten invariants.

\section{Vortices With ZERo AREA AND GENUS}

In this section we construct zero-area gauged Gromov-Witten invariants in genus zero. There are two approaches: in the symplectic approach the moduli space of zero-area vortices $\bar{M}_{n}^{K}\left(\mathbb{P}^{1}, X\right)_{0}$ is constructed via degenerate symplectic reduction on the moduli space of stable maps $\bar{M}_{0, n}\left(\mathbb{P}^{1} \times X\right)$. In the second approach, a moduli stack of zero-area gauged maps $\overline{\mathcal{M}}_{n}^{G}\left(\mathbb{P}^{1}, X\right)_{0}$ is constructed via a stability condition Definition 3.5 for $\overline{\mathcal{M}}_{0, n}\left(\mathbb{P}^{1} \times X\right)$.

3.1. Zero-area vortices. Let $X$ be a compact Hamiltonian $K$-manifold with moment map $\Phi: X \rightarrow \mathfrak{k}^{\vee} \cong \mathfrak{k}$ equipped with an invariant compatible almost complex structure $J$. Let $\operatorname{Map}\left(\mathbb{P}^{1}, X\right)$ denote the space of smooth maps from $\mathbb{P}^{1}$ to $X$. Define

$$
\phi: \operatorname{Map}\left(\mathbb{P}^{1}, X\right) \rightarrow \mathfrak{k}, \quad \phi(u)=\int_{\mathbb{P}^{1}} u^{*} \Phi \operatorname{Vol}_{\mathbb{P}^{1}} .
$$

Definition 3.1. A zero-area-vortex is a map $u \in \operatorname{Map}\left(\mathbb{P}^{1}, X\right)$ satisfying the equations $\phi(u)=0$ and $\bar{\partial} u=0$. An isomorphism of zero-area vortices $u_{0}, u_{1}$ is an element $k \in K$ with $k u_{0}=u_{1}$. A zero-area vortex is stable if it has finite automorphism group.

Let $M^{K}\left(\mathbb{P}^{1}, X, d\right)_{0}$ be the moduli space of isomorphism classes of zero-area vortices of homology class $d \in H_{2}(X)$ :

$$
M^{K}\left(\mathbb{P}^{1}, X, d\right)_{0}=\left\{u \in \operatorname{Map}\left(\mathbb{P}^{1}, X\right) \mid \bar{\partial} u=0, \phi(u)=0, u_{*}\left[\mathbb{P}^{1}\right]=d\right\} / K .
$$

Since any flat connection on $\mathbb{P}^{1} \times K$ is gauge equivalent to the trivial one and has automorphism group isomorphic to $K$, the space $M^{K}\left(\mathbb{P}^{1}, X, d\right)$ may be viewed a 
moduli space of isomorphism classes of pairs $(A, u)$ with $F_{A}=0, \bar{\partial}_{A} u=0$; this justifies the use of the term vortex. For $d \in H_{2}^{K}(X, \mathbb{Z})$ we define $M^{K}\left(\mathbb{P}^{1}, X, d\right)_{0}$ to be the union of components $M^{K}\left(\mathbb{P}^{1}, X, d^{\prime}\right)_{0}$ where $d^{\prime} \in H_{2}(X, \mathbb{Z})$ maps to $d$ under the inclusion of the fiber $X \rightarrow E K \times_{K} X$.

The formal tangent space to $M^{K}\left(\mathbb{P}^{1}, X\right)_{0}$ at any point $[u]$ may be identified with the kernel of a Fredholm operator, as follows. Define

$$
\begin{gathered}
E_{u}: \Omega^{0}\left(\mathbb{P}^{1}, u^{*} T X\right) \rightarrow \mathfrak{k}, \quad v \mapsto \int_{\mathbb{P}^{1}} L_{v} \Phi \operatorname{Vol}_{\mathbb{P}^{1}} \\
E_{u}^{*}: \Omega^{0}\left(\mathbb{P}^{1}, u^{*} T X\right) \rightarrow \mathfrak{k}, \quad v \mapsto \int_{\mathbb{P}^{1}} L_{J v} \Phi \operatorname{Vol}_{\mathbb{P}^{1}} .
\end{gathered}
$$

Thus $E_{u}(v)$ resp. $E_{u}^{*}(v)$ is the derivative of the average of the moment map with respect to $v$ resp. $J v$. Then $M^{K}\left(\mathbb{P}^{1}, X\right)_{0}$ is locally homeomorphic to the zero set of

$$
\begin{aligned}
& \mathcal{F}_{u}^{0}: \Omega^{0}\left(\mathbb{P}^{1}, u^{*} T^{\text {vert }} P(X)\right) \rightarrow(\mathfrak{k} \oplus \mathfrak{k}) \oplus \Omega^{0,1}\left(\mathbb{P}^{1}, u^{*} T^{\text {vert }} P(X)\right) \\
& v \mapsto\left(\int_{\mathbb{P}^{1}} \exp _{u}(v)^{*} P(\Phi), E_{u}^{*} v, \Psi_{u}(v)^{-1} \bar{\partial} \exp _{u}(v)\right)
\end{aligned}
$$

where $\Psi_{u}(v)^{-1}$ is parallel transport from $\exp _{u}(v)$ to $u$, using a Hermitian connection.

Definition 3.2. The linearized operator for a zero-area vortex $u$ is the linearization of $\mathcal{F}_{u}^{0}$,

$$
\tilde{D}_{u}^{0}: \Omega^{0}\left(\mathbb{P}^{1}, u^{*} T X\right) \rightarrow(\mathfrak{k} \oplus \mathfrak{k}) \oplus \Omega^{0,1}\left(\mathbb{P}^{1}, u^{*} T X\right), \quad v \mapsto\left(E_{u} v, E_{u}^{*} v, D_{u} v\right) .
$$

where $D_{u}$ is the standard linearized Cauchy-Riemann operator. We say that a zeroarea-vortex $u$ is regular iff the operator $\tilde{D}_{u}^{0}$ is surjective. The space of infinitesimal deformations of $u$ is $\operatorname{Def}(u)=\operatorname{ker}\left(\tilde{D}_{u}^{0}\right)$.

Let $M^{K, \text { reg }}\left(\mathbb{P}^{1}, X\right)_{0} \subset M^{K}\left(\mathbb{P}^{1}, X\right)_{0}$ denote the locus of regular zero-area vortices. It has the structure of a smooth, finite-dimensional orbifold; the proof is similar to that for pseudoholomorphic maps and will be omitted.

3.2. Compactification. The moduli space $M^{K}\left(\mathbb{P}^{1}, X, d\right)_{0}$ admits a compactification by allowing bubbling in the fibers, as in the case of finite vortex parameter discussed in Theorem 2.7. One way of constructing this compactification is by the following quotient construction as a formal symplectic quotient of the moduli space of stable maps. Let $\bar{M}_{0, n}\left(\mathbb{P}^{1} \times X,(1, d)\right)$ denote the moduli space of parametrized genus 0 stable holomorphic maps $u=\left(u_{1}, u_{2}\right): C \rightarrow \mathbb{P}^{1} \times X$ of homology class $(1, d)$. Define

$$
\phi: \bar{M}_{0, n}\left(\mathbb{P}^{1} \times X,(1, d)\right) \rightarrow \mathfrak{k}, \quad u \mapsto \int_{C} u_{2}^{*} \Phi u_{1}^{*} \operatorname{Vol}_{\mathbb{P}^{1}}
$$

The map $\phi$ can be considered as a moment map for the action of $K$ on $\bar{M}_{0, n}\left(\mathbb{P}^{1} \times\right.$ $X,(1, d))$ for a closed two-form given by integrating the pull-back of $\omega \in \Omega^{2}(X)$ over 
the principal component as in (4). By definition of zero-area vortices there is an isomorphism

$$
\bar{M}_{n}^{K}\left(\mathbb{P}^{1}, X, d\right)_{0} \cong \phi^{-1}(0) / K=: \bar{M}_{0, n}\left(\mathbb{P}^{1} \times X,(1, d)\right) / / K .
$$

The local structure of the regular locus is described as follows:

Definition 3.3. Let $u: C \rightarrow \mathbb{P}^{1} \times X$ be a zero-area vortex with combinatorial type $\Gamma$. The linearized operator for $u$ is

$\tilde{D}_{u}^{0}: \Omega^{0}\left(C, u^{*} T\left(\mathbb{P}^{1} \times X\right)\right) \rightarrow(\mathfrak{k} \oplus \mathfrak{k}) \oplus \Omega^{0,1}\left(\tilde{C}, u^{*} T\left(\mathbb{P}^{1} \times X\right)\right), \quad v \mapsto\left(E_{u} v, E_{u}^{*} v, D_{u} v\right)$

where $D_{u}$ is the usual linearized Cauchy-Riemann operator on $\Omega^{0}\left(C, u^{*} T X\right), E_{u}, E_{u}^{*}$ are the operators on the principal component and $\tilde{C}$ is the normalization of $C$. We say that $u$ is regular iff $\tilde{D}_{u}^{0}$ is surjective. The space of infinitesimal deformations of constant type of $u$ is

$$
\operatorname{Def}_{\Gamma}(u)=\operatorname{ker}\left(\tilde{D}_{u}^{0}\right) / \operatorname{aut}(C) .
$$

The space of infinitesimal deformations of $u$ is the space of infinitesimal deformations of fixed type, plus the space of gluing parameters:

$$
\operatorname{Def}(u)=\operatorname{Def}_{\Gamma}(u) \oplus \bigoplus_{i=1}^{m} T_{w_{i}^{+}} C \otimes T_{w_{i}^{-}} C
$$

where $m$ is the number of nodes of $C$.

Theorem 3.4. For any $c>0$, the union over $d \in H_{2}^{K}(X, \mathbb{Z})$ with $\left\langle\left[\omega_{K}\right], d\right\rangle<c$ of $\bar{M}_{n}^{K}\left(\mathbb{P}^{1}, X, d\right)_{0}$ is a compact, Hausdorff space. The regular locus $\bar{M}_{n}^{K, \text { reg }}\left(\mathbb{P}^{1}, X\right)_{0}$ admits the structure of a stratified-smooth topological orbifold, with tangent space at $[u]$ isomorphic to $\operatorname{Def}(u)$.

Proof. This follows from the symplectic quotient description (13) and compactness and regularity properties of the moduli space of stable maps.

3.3. Zero-semistability. In this section we give an algebraic interpretation of the moduli space of zero-area vortices in terms of a stability condition. Let $X$ be a smooth projectively-embedded $G$-variety. The $G$-action on $X$ induces an action on the stack $\overline{\mathcal{M}}_{0, n}\left(\mathbb{P}^{1} \times X,(1, d)\right)$ by translation, see Romagny 33. for discussion of group actions on stacks.

Definition 3.5. Given a non-zero element $\lambda \in \mathfrak{k}$ and a stable map $u: C \rightarrow \mathbb{P}^{1} \times X$, the associated graded map $\operatorname{Gr}(u)$ is the Gromov limit of maps $\exp (t i \lambda) u$ as $t \rightarrow-\infty$. The degree of $\lambda$ with respect to $u$ is $\mu_{\lambda}(u):=\left\langle\operatorname{Gr}(u)_{0}^{*} \Phi, \lambda\right\rangle$, where the pairing is taken at any point in the principal component. A stable map $u: C \rightarrow \mathbb{P}^{1} \times X$ is 0 semistable iff $\mu_{\lambda}(u) \leq 0$ for all $\lambda \in \mathfrak{g}, 0$-unstable iff it is not 0 -semistable, and 0-stable iff it is 0 -semistable with strict inequality for each non-zero $\lambda$. An isomorphism of 0-semistable maps $u: C \rightarrow \mathbb{P}^{1} \times X, u^{\prime}: C^{\prime} \rightarrow \mathbb{P}^{1} \times X$ is an isomorphism $\psi: C \rightarrow C^{\prime}$ and an element $g \in G$ such that $g u=u^{\prime} \circ \psi$. 
A stable map is 0 -unstable iff a generic value on the principal component is 0 unstable for some one-parameter subgroup, which is a closed condition. Hence the 0semistable locus is open in $\overline{\mathcal{M}}_{0, n}\left(\mathbb{P}^{1} \times X\right)$. (There is a seemingly unavoidable conflict between stability terminology here: a stable map may or may not be 0-(semi)stable.) We denote by $\overline{\mathcal{M}}_{n}^{G}\left(\mathbb{P}^{1}, X\right)_{0}$ the stack of 0 -semistable maps to $X$. More explicitly $\overline{\mathcal{M}}_{n}^{G}\left(\mathbb{P}^{1}, X\right)_{0}$ is a substack of the quotient stack $\overline{\mathcal{M}}_{0, n}\left(\mathbb{P}^{1} \times X\right) / G$ of $\overline{\mathcal{M}}_{0, n}\left(\mathbb{P}^{1} \times X\right)$ by $G$. An object of $\overline{\mathcal{M}}_{0, n}\left(\mathbb{P}^{1} \times X\right) / G$ over a scheme $S$ consists a principal $G$-bundle $P \rightarrow S$ and a stable map $u: C \rightarrow \mathbb{P}^{1} \times P(X)$ over $S$. Artin charts for $\overline{\mathcal{M}}_{0, n}\left(\mathbb{P}^{1} \times X\right) / G$ are induced by charts for $\overline{\mathcal{M}}_{0, n}\left(\mathbb{P}^{1} \times X\right)$, making $\overline{\mathcal{M}}_{n}^{G}\left(\mathbb{P}^{1}, X\right)_{0} \subset \overline{\mathcal{M}}_{0, n}\left(\mathbb{P}^{1} \times X\right) / G$ into an Artin stack cf. [33], [19, Appendix].

Theorem 3.6. Suppose that every 0-semistable gauged map is stable. For any constant $c>0$, the union of components $\overline{\mathcal{M}}_{n}^{G}\left(\mathbb{P}^{1}, X, d\right)_{0}$ with homology class $d \in$ $H_{2}(X, \mathbb{Z})$ satisfying $\langle d,[\omega]\rangle<c$ is a proper Deligne-Mumford stack with a relative perfect obstruction theory over $\overline{\mathfrak{M}}_{n}\left(\mathbb{P}^{1}\right)$.

To show properness of $\overline{\mathcal{M}}_{n}^{G}\left(\mathbb{P}^{1}, X\right)_{0}$ we use the following result, similar to Kirwan 22 and Ness [30, which gives an equivalence between the algebro-geometric and symplecto-geometric definitions. We say that $\lambda \in \mathfrak{k}$ is a maximally destabilizing vector iff for any $\xi \in \mathfrak{k}, \mu_{\xi}(u) /\|\xi\| \leq \mu_{\lambda}(u) /\|\lambda\|$ with equality iff $\mathbb{R}_{>0} \xi=\mathbb{R}_{>0} \lambda$.

Proposition 3.7. Let $u: C \rightarrow \mathbb{P}^{1} \times X$ be a stable map. Then

(a) the map $u$ is 0 -semistable iff $\overline{G u} \cap \phi^{-1}(0) \neq \emptyset$;

(b) $G u \cap \phi^{-1}(0)$ contains at most one $K$-orbit;

(c) Any 0-unstable $u$ has a maximally destabilizing vector $\lambda$.

Proof. In Kempf-Ness [21] and Ness [30] the case of projective or Kähler actions was considered. The case of degenerate polarizations, when the 2-form is only required to be non-degenerate on each orbit, is discussed in [42, Remark 7.2.5].

Remark 3.8. The theory of the Jordan-Hölder vector is missing. That is, we do not know whether, if $\overline{G u}$ contains a vortex, whether the orbit of such a vortex is unique.

Proposition 3.9. Suppose that every 0-area vortex has finite automorphism group. Then the coarse moduli space of $\overline{\mathcal{M}}_{n}^{G}\left(\mathbb{P}^{1}, X\right)_{0}$ is homeomorphic to the moduli space of 0-area vortices $\bar{M}_{n}^{K}\left(\mathbb{P}^{1}, X\right)_{0}$.

Proof. The assumption on finite automorphism group implies stable $=$ semistable, since the associated graded of any 0 -semistable but not 0 -stable has infinite automorphism group. By part (a) of 3.7, any 0-stable map $u$ is complex orbit equivalent to a 0-area vortex; the resulting map from the coarse moduli space (the quotient of the semistable locus by the action) has a continuous inverse given by the inclusion of $\phi^{-1}(0)$ into the 0 -stable locus. 
Proof of Theorem 3.6. Properness follows from properness of the coarse moduli space in Theorem 3.4; the case of non-zero vortex parameter is discussed in [14]. A relative perfect obstruction theory is given by $R p_{*}\left(e^{*} T(X / G)\right)^{\vee}$ as in Behrend [1] and the canonical map to the cotangent complex, where $p$ is the universal curve and $e$ the universal morphism, c.f. Gonzalez-Woodward [14, Theorem 4.23].

3.4. Zero-area gauged Gromov-Witten invariants. Suppose that $X$ is a smooth projectively-embedded $G$-variety. Given $u: C \rightarrow \mathbb{P}^{1} \times X$ we denote by $u_{1}: C \rightarrow \mathbb{P}^{1}$ the component with values in $\mathbb{P}^{1}$.

Lemma 3.10. There exists a forgetful morphism $f: \overline{\mathcal{M}}_{n}^{G}\left(\mathbb{P}^{1}, X\right)_{0} \rightarrow \overline{\mathcal{M}}_{n}\left(\mathbb{P}^{1}\right)$ which maps $(P, C, u, \underline{z})$ to the stable map obtained from $\left(C, u_{1}, \underline{z}\right)$ by collapsing unstable components.

Proof. The existence of a morphism $\overline{\mathcal{M}}_{0, n}\left(\mathbb{P}^{1} \times X\right) \rightarrow \overline{\mathcal{M}}_{0, n}\left(\mathbb{P}^{1}\right)$ collapsing unstable components follows from the functoriality properties of the moduli space of stable maps given in Behrend-Manin [3]. The restriction to the 0-semistable locus is invariant and so factors through the quotient $\overline{\mathcal{M}}_{n}^{G}\left(\mathbb{P}^{1}, X\right)_{0}$.

Let ev $: \overline{\mathcal{M}}_{n}^{G}\left(\mathbb{P}^{1}, X\right)_{0} \rightarrow(X / G)^{n}$ denote the evaluation map at the marked points. Let ev* denote the induced pull-back in rational cohomology of coarse moduli spaces $\mathrm{ev}^{*}: H_{G}(X, \mathbb{Q})^{n} \rightarrow H\left(\bar{M}_{n}^{G}\left(\mathbb{P}^{1}, X\right)_{0}, \mathbb{Q}\right)$.

Definition 3.11. Suppose that every semistable zero-area gauged map of class $d \in H_{2}(X, \mathbb{Z})$ has finite automorphism group. The zero-area gauged Gromov-Witten invariant for $\alpha \in H_{G}(X, \mathbb{Q})^{n}, \beta \in H\left(\bar{M}_{n}\left(\mathbb{P}^{1}\right), \mathbb{Q}\right), d \in H_{2}^{G}(X, \mathbb{Z})$ is

$$
\langle\alpha ; \beta\rangle_{d, 0}=\int_{\left[\overline{\mathcal{M}}_{n}^{G}\left(\mathbb{P}^{1}, X, d\right)_{0}\right]} \mathrm{ev}^{*} \alpha \cup f^{*} \beta \in \mathbb{Q} .
$$

From the symplectic point of view, the invariants are defined without virtual fundamental cycles if every vortex is regular, by integration using the usual orbifold fundamental class. At least in principle, the zero-area gauged Gromov-Witten invariants may be computed from the usual equivariant Gromov-Witten invariants using localization theorems in [18, [41].

The splitting axiom for the zero-area gauged Gromov-Witten invariants is the same as that for finite vortex parameter discussed in [14], that is, they form a cohomological trace on the cohomological field theory defined by the equivariant Gromov-Witten invariants introduced by Givental in $[13$. We will not prove the splitting axiom here; it follows from the equality with the gauged Gromov-Witten invariants for small area proved later. 


\section{THE ZERO-AREA LIMIT}

In this section we give both an algebraic and symplectic study of the moduli spaces in the zero-area limit.

\subsection{Small-area limit of Mundet-stability.}

Theorem 4.1. Let $X$ be a smooth projectively-embedded $G$-variety. There exists a $\rho_{0}>0$ such that for $\rho<\rho_{0}$, any gauged map $(P, C, u)$ of genus zero is $\rho$-semistable iff $P$ is trivializable and (after identifying $P \rightarrow \mathbb{P}^{1} \times G$ so that $P(X) \cong \mathbb{P}^{1} \times X$ ) the map $u$ is 0-semistable.

Proof. For the proof we use the definition of degree (11) using the moment map. Let $\rho_{0}>0$ be such that $\sup _{x \in X} \rho_{0}\|\Phi(x)\| \int_{\mathbb{P}^{1}} \operatorname{Vol}_{\mathbb{P}^{1}}<1$. Since the first Chern number of any line bundle is integral, a pair $(\sigma, \lambda)$ in (10) violates semistability for $\rho<\rho_{0}$ iff

$$
\mu_{\sigma, \lambda}(P, u)=\int_{\mathbb{P}^{1}} c_{1}\left(p_{*} \sigma^{*} P \times_{L} \mathbb{C}_{\lambda}\right)+\rho\left\langle P(\Phi) \circ \operatorname{Gr}(u)_{0}, \lambda\right\rangle \operatorname{Vol}_{\mathbb{P}^{1}}>0
$$

iff $\int_{\sigma} c_{1}\left(p_{*} \sigma^{*} P \times_{L} \mathbb{C}_{\lambda}\right) \geq 0$ and if equality holds then

$$
\left\langle P(\Phi) \circ \operatorname{Gr}(u)_{0}, \lambda\right\rangle>0 .
$$

It follows that $(P, C, u)$ is $\rho$-semistable iff $P$ is semistable, hence trivial (since $\mathbb{P}^{1}$ has genus zero), and $u$ satisfies the 0 -semistability condition of Definition 3.5 .

Corollary 4.2. There exists a $\rho_{0}>0$ such that for $\rho<\rho_{0}$, there is an equivalence from $\overline{\mathcal{M}}_{n}^{G}\left(\mathbb{P}^{1}, X\right)_{0}$ to $\overline{\mathcal{M}}_{n}^{G}\left(\mathbb{P}^{1}, X\right)_{\rho}$ of Deligne-Mumford stacks equipped with relative perfect obstruction theories.

Proof. Theorem 4.1 gives an equivalence from $\overline{\mathcal{M}}_{n}^{G}\left(\mathbb{P}^{1}, X\right)_{0}$ to $\overline{\mathcal{M}}_{n}^{G}\left(\mathbb{P}^{1}, X\right)_{\rho}$ for $\rho$ as in the Theorem. The relative perfect obstruction theories are equivalent, by definition.

Theorem 1.2 of the Introduction follows.

4.2. Small-area limit of vortices. In this section we consider the small-area limit in the case that the target is a Hamiltonian $K$-manifold, in which we lack a holomorphic description of the moduli space. First we prove a result in the case without bubbling:

Theorem 4.3. Suppose that $\left(A_{\nu}, u_{\nu}\right)$ is a sequence of $\rho_{\nu}$ vortices of constant homology class $d \in H_{2}^{K}(X)$, with $\rho_{\nu} \rightarrow 0$. If $c_{\nu}=\sup \left|d_{A_{\nu}} u_{\nu}\right|$ is bounded, then after passing to a subsequence, there exists a sequence of gauge transformations $k_{\nu} \in \mathcal{K}(P)$ and a zero-area-vortex $u_{\infty}$ such that $k_{\nu} A_{\nu}$ converges to a trivial connection $A_{\infty}$ and (using the trivialization induced by $A_{\infty}$ ) $k_{\nu} u_{\nu} \rightarrow u_{\infty}$ uniformly in all derivatives. 
The proof relies on the following well-known lemma, cf. [8, Lemma 2.3.10], which controls the norm of the connection linearly in terms of the norm of the curvature in genus zero:

Lemma 4.4. Let $p \geq 1$. There exist constants $\delta>0, c>0$ such that if $A$ is a connection on the trivial bundle over $\mathbb{P}^{1}$ in Coulomb gauge with respect to the trivial connection, that is, $\mathrm{d}^{*} A=0$, then

$$
\|A\|_{1, p}<\delta \Longrightarrow\|A\|_{1, p} \leq c\left\|F_{A}\right\|_{0, p} .
$$

Proof. Using the elliptic estimate for $\mathrm{d}+\mathrm{d}^{*}$ and $H^{1}\left(\mathbb{P}^{1}\right)=0$ we obtain $\|A\|_{1, p} \leq$ $c_{1}\|\mathrm{~d} A\|_{0, p}$. The Sobolev multiplication estimate for $[A, A] / 2$ gives

$$
\|\mathrm{d} A\|_{0, p} \leq c_{2}\left(\left\|F_{A}\right\|_{0, p}+\|A\|_{1, p}^{2}\right),
$$

and thus for some $\delta, c>0$ we have $\|A\|_{1, p} \leq c\left\|F_{A}\right\|_{0, p}$ if $\|A\|_{1, p}<\delta$.

Proof of Theorem 4.3. Since $X$ is compact, the curvature of $A_{\nu}$ goes to zero as $\nu \rightarrow 0$ in $0, p$ norm,

$$
\left\|F_{A_{\nu}}\right\|_{0, p}^{p} \leq \rho_{\nu} \sup _{x \in X}|\Phi(x)|^{p} \int_{\mathbb{P}^{1}} \operatorname{Vol}_{\mathbb{P}^{1}} \rightarrow 0
$$

using the vortex equation in Definition 2.1, By weak Uhlenbeck compactness, see e.g. [40], after gauge transformation and passing to a subsequence $A_{\nu}$ converges weakly in $W^{1, p}$ to a connection $A_{\infty}$ for some $p>2$. Then $F_{A_{\nu}}$ converges weakly in $L^{p}$ to $F_{A_{\infty}}$. Since $F_{A_{\nu}}$ converges to 0 in $L^{p}$ the convergence is strong and $F_{A_{\infty}}$ vanishes. After $W^{2, p}$ gauge transformation, we may assume that $A_{\infty}$ is smooth. Parallel transport by $A_{\infty}$ defines a trivialization of $P$, since $\mathbb{P}^{1}$ is simply-connected. Then $u_{\nu}$ defines a map to $X$ and $A_{\nu}$ resp. $F_{A_{\nu}}$ defines a one-form resp. two-form with values in the Lie algebra, for which we use the same notation. After gauge transformation, we may assume $\mathrm{d}^{*} A_{\nu}=0$. By Lemma $4.4\left\|A_{\nu}\right\|_{1, p}<c \rho_{\nu}$, in particular, $A_{\nu}$ converges to 0 in $C^{0}$ norm. Integrating the vortex equation (1) gives

$$
\int_{\mathbb{P}^{1}} u_{\nu}^{*} \Phi \operatorname{Vol}_{\mathbb{P}^{1}}=\rho_{\nu}^{-1} \int_{\mathbb{P}^{1}} \frac{1}{2}\left[A_{\nu}, A_{\nu}\right] \operatorname{Vol}_{\mathbb{P}^{1}} \rightarrow 0 .
$$

Since $\sup \left|d_{A_{\nu}} u_{\nu}\right|$ is bounded, $u_{\nu}$ converges in $C^{0}$ to a section $u_{\infty}$ with

$$
\int_{\mathbb{P}^{1}} u_{\infty}^{*} \Phi \operatorname{Vol}_{\mathbb{P}^{1}}=\lim _{\nu \rightarrow \infty} \int_{\mathbb{P}^{1}} u_{\nu}^{*} \Phi \operatorname{Vol}_{\mathbb{P}^{1}}=0 .
$$

One obtains uniform convergence for $A_{\nu}, u_{\nu}$ in all derivatives and holomorphicity of $u_{\infty}$ by elliptic bootstrapping as in [5, Section 3].

More generally for polystable vortices we have a similar result Theorem 1.1.

Proof of Theorem 1.1. As in the proof of Theorem 4.3, after gauge transformation and passing to a subsequence we may assume that $A_{\nu}$ converges in $C^{0}$ to a flat connection $A_{\infty}$, which defines a trivialization of $P$. On any set on which the first derivative is bounded, the principal component of $u_{\nu}$ converges to a holomorphic map to $X$ and $A_{\nu}$ converges to $A_{\infty}$ uniformly in all derivatives as before. Gromov 
convergence of $u_{\nu}$ to a stable map $u_{\infty}$ follows by Ott's arguments in [31, using the fact that $\rho_{\nu} \rightarrow 0$ implies that the bounds [31, (2.25)] are satisfied. In particular, if $u_{\nu, 0}$ resp $u_{\infty, 0}$ denotes the principal component then $u_{\nu, 0}$ converges to $u_{\infty, 0}$ uniformly in all derivatives on compact subsets of the complement of the (finite) bubbling set $Z$. Integrating the vortex equation gives

$$
\begin{aligned}
\left\|\int_{\mathbb{P}^{1}} u_{\infty, 0}^{*} \Phi\right\| & =\lim _{\epsilon \rightarrow 0} \lim _{\nu \rightarrow \infty}\left\|\int_{\mathbb{P}^{1}-B_{\epsilon}(Z)} u_{\nu, 0}^{*} \Phi\right\| \\
& \leq \lim _{\epsilon \rightarrow 0} \lim _{\nu \rightarrow \infty}\left\|\int_{\mathbb{P}^{1}} u_{\nu, 0}^{*} \Phi\right\|+\operatorname{Vol}\left(B_{\epsilon}(Z)\right) \sup (\|\Phi\|) \\
& \leq \lim _{\nu \rightarrow \infty} c \rho_{\nu}=0
\end{aligned}
$$

where $B_{\epsilon}(Z)$ denotes the union of $\epsilon$-balls around the points in $Z$.

\section{Application to abelianization}

In this section we prove the abelianization Theorem 1.3 for gauged GromovWitten invariants of zero area, hence for gauged Gromov-Witten invariants of small area. We first give a topological proof, assuming that $X$ is convex (that is, every pseudoholomorphic map is regular) in which case the proof is essentially the same as Martin [26]. Suppose that $K$ is a compact connected Lie group. Let $T \subset K$ denote a maximal torus and $W=N(T) / T$ its Weyl group. Consider the map $H^{T}(X, \mathbb{Z}) \rightarrow$ $H^{K}(X, \mathbb{Z})$ induced by the inclusion $T \rightarrow K$. We write $d_{T} \mapsto d_{K}$ if $d_{T} \in H_{2}^{T}(X, \mathbb{Z})$ maps to $d_{K} \in H_{2}^{K}(X, \mathbb{Z})$ under the isomorphism $H_{2}^{T}(X, \mathbb{Z}) \rightarrow H_{2}^{K}(X, \mathbb{Z})$ induced by $X \times_{T} E T \rightarrow X \times_{K} E K$. We denote by $\langle\alpha, \beta\rangle_{K, d_{K}, \rho}$ resp. $\langle\alpha, \beta\rangle_{T, d_{T}, \rho}^{t w i s t}$ the untwisted resp. $\mathfrak{g} / \mathfrak{t}$-twisted vortex invariants for $K$ resp. $T$.

Proof of (2) in the convex case. Suppose that $d_{K} \in H_{2}^{K}(X, \mathbb{Z})$ is the image of $d \in$ $H_{2}(X, \mathbb{Z})$, and $d_{T}$ is the image of $d$ in $H_{2}^{T}(X, \mathbb{Z})$. Let $\phi_{T}^{-1}(0)$ resp. $\phi_{K}^{-1}(0)$ denote the subspace of stable maps of homology class $d$ with average $T$ resp. $K$ moment map equal to zero. The projection $\phi_{K}^{-1}(0) / T \rightarrow \phi_{K}^{-1}(0) / K$ has fiber $K / T$ with Euler characteristic $\chi(K / T)=\chi\left((K / T)^{T}\right)=\# W$. The inclusion $\phi_{K}^{-1}(0) / T \rightarrow \phi_{T}^{-1}(0) / T$ has normal bundle $\phi_{K}^{-1}(0) \times_{T}(\mathfrak{k} / \mathfrak{t})^{\vee}$ with transverse section induced by $\phi_{K}$. Since the connections are trivial in this case, the index of $(\mathfrak{k} / \mathfrak{t})^{\vee}$ is $(\mathfrak{k} / \mathfrak{t})^{\vee}$ itself. Hence

$$
\begin{aligned}
\langle\alpha, \beta\rangle_{K, d_{K}, 0} & =\int_{\phi_{K}^{-1}(0) / K} \operatorname{ev}^{*} \alpha \cup f^{*} \beta \\
& =(\# W)^{-1} \int_{\phi_{K}^{-1}(0) / T} \operatorname{ev}^{*} \alpha \cup f^{*} \beta \cup \operatorname{Eul}(\operatorname{Ind}(\mathfrak{k} / \mathfrak{t})) \\
& =(\# W)^{-1} \int_{\phi_{T}^{-1}(0) / T} \operatorname{ev}^{*} \alpha \cup f^{*} \beta \cup \operatorname{Eul}\left(\operatorname{Ind}\left((\mathfrak{k} / \mathfrak{t}) \oplus(\mathfrak{k} / \mathfrak{t})^{\vee}\right)\right) \\
& =(\# W)^{-1}\langle\alpha, \beta\rangle_{T, d_{T}, 0}^{t w i s t} .
\end{aligned}
$$


For the case when the target an arbitrary smooth projectively-embedded $G$-variety $X$ we derive the abelianization formula (2) from a version for sheaf cohomology. A similar strategy is used for various virtual integration identities in Joshua [20]. Recall from Lee 24] that the virtual structure sheaf of a Deligne-Mumford stack $\mathcal{S}$ equipped with a perfect obstruction theory $\phi: E \rightarrow L_{\mathcal{S}}$ is defined as follows. Let $\mathcal{E}_{\mathcal{S}}=h^{1} / h^{0}\left(E^{\vee}\right)$ denote the corresponding cone stack and $C_{\mathcal{S}}$ the intrinsic normal cone of Behrend-Fantechi [2]. Define

$$
\mathcal{O}_{\mathcal{S}}^{\text {vir }}:=\mathcal{O}_{\mathcal{S}} \bigotimes_{\substack{0_{\mathcal{E}_{\mathcal{S}}}^{-1}\left(\mathcal{O}_{\mathcal{E}_{\mathcal{S}}}\right) \\ L}}^{L} 0_{\mathcal{E}_{\mathcal{S}}}^{-1}\left(\mathcal{O}_{C_{\mathcal{S}}}\right)
$$

where $0_{\mathcal{E}_{\mathcal{S}}}: \mathcal{S} \rightarrow \mathcal{E}_{\mathcal{S}}$ is the inclusion of the vertex. The virtual push-forward of an object $F$ of the derived category is

$$
R f_{*}^{\mathrm{vir}} F:=R f_{*}\left(F \bigotimes_{\mathcal{O}_{\mathcal{S}_{0}}}^{L} \mathcal{O}_{\mathcal{S}_{0}}^{\mathrm{vir}}\right) .
$$

If $\mathcal{S}_{1}$ is a point then we denote by $\chi^{\mathrm{vir}}(F)$ the Euler characteristic of $R f_{*}^{\mathrm{vir}}(F)$.

We have the following equivariant version of abelianization which is fairly trivial. Denote by $\underline{\Lambda}(\mathfrak{g} / \mathfrak{t})$ the vector bundle with fiber the exterior algebra $\Lambda(\mathfrak{g} / \mathfrak{t})$ on $\mathfrak{g} / \mathfrak{t}$.

Proposition 5.1. For any $G$-equivariant vector bundle $E$ on $\overline{\mathcal{M}}_{0, n}\left(\mathbb{P}^{1} \times X,(1, d)\right)$, $\left.\chi^{\operatorname{vir}}(E)^{G}=(\# W)^{-1} \chi^{\operatorname{vir}}(E \otimes \underline{\Lambda}(\mathfrak{g} / \mathfrak{t}))\right)^{T}$.

Proof. For any finite-dimensional representation $V$ of $G$ the $\operatorname{dimension} \operatorname{dim}\left(V^{G}\right)$ of the space of invariants is $(\# W)^{-1} \operatorname{dim}(V \otimes \Lambda(\mathfrak{g} / \mathfrak{t}))^{T}$ by the Weyl character formula. Applying this to the Euler characteristic on the left gives the result.

To derive abelianization from this fact we need the following properties of local cohomology, the second of which uses a bit of derived algebraic geometry; they are versions of the Thom and Gysin isomorphisms in K-theory. Suppose that $f: \mathcal{S}_{0} \rightarrow$ $\mathcal{S}_{1}$ is a locally closed embedding. We denote by $\chi_{\mathcal{S}_{0}}^{\mathrm{vir}}\left(\mathcal{S}_{1}, F\right)$ the Euler characteristic of the local cohomology sheaves

$$
\mathcal{H}_{\mathcal{S}_{0}}^{\mathrm{vir}}\left(\mathcal{S}_{1}, F\right):=\mathcal{H}_{\mathcal{S}_{0}}\left(\mathcal{S}_{1}, F \otimes^{L} \mathcal{O}_{\mathcal{S}_{0}}^{\text {vir }}\right)
$$

By Schürg [34], any perfect obstruction theory arises from a quasi-smooth derived structure. A derived structure on the moduli stack of stable maps, which is the only case we use here, is discussed in [35].

Proposition 5.2. Suppose that $F \rightarrow \mathcal{S}_{0}$ is a vector bundle.

(a) Suppose that $f: \mathcal{S}_{0} \rightarrow \mathcal{S}_{1}$ is a vector bundle. Then $R f_{*}^{\text {vir }} F$ is isomorphic to $\iota^{*} F \otimes \mathrm{S}\left(\mathcal{S}_{1}^{\vee}\right)$ where $\iota: \mathcal{S}_{1} \rightarrow \mathcal{S}_{0}$ is the zero section.

(b) Let $f: \mathcal{S}_{0} \rightarrow \mathcal{S}_{1}$ be a derived regular embedding with normal bundle $\mathcal{N}$. Then the local cohomology sheaf $\mathcal{H}_{\mathcal{S}_{0}}^{\mathrm{vir}}\left(\mathcal{S}_{1}, F\right)$ admits a filtration by order of 
vanishing at $\mathcal{S}_{0}$ with associated graded complex $\left(\mathrm{S}(\mathcal{N}) \otimes \operatorname{det}(\mathcal{N}) \otimes f^{*} F \otimes^{L}\right.$ $\left.\mathcal{O}_{\mathcal{S}_{1}}^{\text {vir }}\right)[\operatorname{rank}(\mathcal{N})]$

The Gysin isomorphism is (for schemes) Grothendieck [16, p.14]. Local cohomology for derived algebraic geometry is developed in Lurie [25], although we are certainly not using the full theory here.

Local cohomology groups appear in the spectral sequence for the Kirwan-Ness stratification for the action of $G$ on $\overline{\mathcal{M}}:=\overline{\mathcal{M}}_{0, n}\left(\mathbb{P}^{1} \times X,(1, d)\right)$. Recall first the Kirwan-Ness stratification for the action of $G$ on $X$ : Any non-zero $\lambda \in \mathfrak{k}$ defines a parabolic subgroup $R_{\lambda}$, the set of elements $r \in R$ such that $\operatorname{Ad}(\exp (z i \lambda)) r$ has a limit as $z \rightarrow-\infty$. The centralizer $G_{\lambda}$ of $\lambda$ is a Levi subgroup and the map $r \rightarrow \lim _{z \rightarrow 0} \operatorname{Ad}(\exp (z \lambda)) r$ is the quotient by a maximal unipotent, and so a group homomorphism. Let $X=\bigcup_{\lambda} X_{\lambda}$ denote the Kirwan-Ness stratification of $X$; here $\lambda$ ranges over equivalence classes of one-parameter subgroups. For each $\lambda$, let $Z_{\lambda}$ denote the corresponding fixed point set of the one-parameter subgroup generated by $\lambda$. Let $Z_{\lambda}^{\text {ss }}$ the semistable locus for the action of $G_{\lambda} / \mathbb{C}_{\lambda}^{*}$ on $Z_{\lambda}, Y_{\lambda}$ the subset of $X$ flowing to $Z_{\lambda}$ under $\exp (z \lambda)$, and $Y_{\lambda}^{\text {ss }}$ the inverse image of $Z_{\lambda}^{\text {ss }}$. Then (see [22], [30]) $X_{\lambda}=G \times{ }_{R_{\lambda}} Y_{\lambda}^{\mathrm{ss}}$.

Strictly speaking, there is no Kirwan-Ness stratification for the $G$-action on $\overline{\mathcal{M}}$ since there is no polarization. However, the stack $\overline{\mathcal{M}}$ has a similar stratification given as follows.

Definition 5.3. Let $\mathcal{M}^{\lambda}$ denote the substack of $\overline{\mathcal{M}}$ consisting of morphisms $u$ whose maximally destabilizing vector is conjugate to $\lambda$. Let $\mathcal{Z}^{\lambda}$ denote the substack of maps that are fixed by $\mathbb{C}_{\lambda}^{*}$ whose principal component takes values in $Z_{\lambda} ; \mathcal{Z}^{\lambda \text {,ss }}$ the locus in $\mathcal{Z}^{\lambda}$ of 0 -semistable maps for the residual action of $G_{\lambda} / \mathbb{C}_{\lambda}^{*} ; \mathcal{Y}^{\lambda}$ (resp. $\left.\mathcal{Y}^{\lambda, \mathrm{ss}}\right)$ denote the substack of maps $u$ that flow under $\exp (z \lambda)$ to $\mathcal{Z}^{\lambda}$ (resp. $\mathcal{Z}^{\lambda, \mathrm{ss}}$ ).

Proposition 5.4. Each $\mathcal{M}^{\lambda}$ is a locally closed substack of $\overline{\mathcal{M}}$ equal to $G \times_{G_{\lambda}} \mathcal{Y}^{\lambda \text {,ss }}$. The closure of $\mathcal{M}^{\lambda}$ is contained in the union of $\mathcal{M}^{\nu}$ with $\|\nu\| \geq\|\lambda\|$. The embedding $\mathcal{M}^{\lambda} \rightarrow \overline{\mathcal{M}}$ is the truncation of a regular embedding of quasismooth derived stacks.

Proof. That $\mathcal{M}^{\lambda}$ is locally closed follows from the fact that the Hilbert-Mumford weight for $u$ with respect to $\lambda$ is given by the weight for a generic value $u(z), z \in C_{0}$. The equality $\mathcal{M}^{\lambda}=G \times_{G_{\lambda}} \mathcal{Y}^{\lambda \text {,ss }}$ would follow from Kirwan [22] applied to the coarse moduli space $\bar{M}$ of $\overline{\mathcal{M}}$, except that $\bar{M}$ is singular and has possibly degenerate two-form, where smooth. However, each $G$ orbit in $\bar{M}$ is smooth and has a nondegenerate Kähler form, and [42, Remark 7.2.5] shows that the theory of maximally destabilizing vectors extends to this degenerate case. That the closure of $\mathcal{M}^{\lambda}$ is contained in the union of $\mathcal{M}^{\nu}$ with $\|\nu\| \leq\|\lambda\|$ follows from the description of the norm $\|\lambda\|$ of the maximally destabilizing vector $\lambda$ for $u$ as the infimum of $\|\phi\|$ over the orbit $G u$, see [42, Lemma 5.4.3]. Regularity of $\mathcal{M}^{\lambda} \rightarrow \overline{\mathcal{M}}$ follows from embedding $X \rightarrow \mathbb{P}^{N}$ which induces an embedding of $\overline{\mathcal{M}}_{0, n}\left(\mathbb{P}^{1} \times X\right)$ into the smooth stack $\overline{\mathcal{M}}_{0, n}\left(\mathbb{P}^{1} \times \mathbb{P}^{N}\right)$, for which the strata are honestly smooth. 
The following is a version of non-abelian localization for the action of $G$ on the stack $\overline{\mathcal{M}}$, compare [36].

Theorem 5.5. For $G$-equivariant vector bundle $V$ on $\overline{\mathcal{M}}$ we have

$$
\begin{aligned}
\chi^{\mathrm{vir}}(\overline{\mathcal{M}}, V)^{G}= & \sum_{\lambda}(-1)^{\operatorname{codim}\left(\mathcal{M}^{\lambda}\right)} \chi^{\mathrm{vir}}\left(\mathcal{Z}^{\lambda, \mathrm{ss}}, V \otimes\right. \\
\quad & \left.\quad \mathrm{S}\left(T_{\mathcal{Z}^{\lambda}}\left(\mathcal{Y}^{\lambda}\right)^{\vee} \oplus T_{\mathcal{M}^{\lambda}} \overline{\mathcal{M}}\right) \otimes \operatorname{det}\left(T_{\mathcal{M}^{\lambda}} \overline{\mathcal{M}}\right) \otimes \underline{\Lambda}\left(\mathfrak{g} / \mathfrak{r}_{\lambda}\right)\right)^{G_{\lambda}}
\end{aligned}
$$

where we have omitted restrictions to simplify notation.

Proof. As in Teleman [36]. By the spectral sequence associated to the stratification we have

$$
\chi^{\operatorname{vir}}(\overline{\mathcal{M}}, V)^{G}=\sum_{\lambda}(-1)^{\operatorname{codim}\left(\mathcal{M}^{\lambda}\right)} \chi_{\mathcal{M}^{\lambda}}^{\operatorname{vir}}(\overline{\mathcal{M}}, V)^{G} .
$$

Using the Gysin isomorphism 5.2 (b),

$$
\chi_{\mathcal{M}^{\lambda}}^{\operatorname{vir}}(\overline{\mathcal{M}}, V)^{G}=(-1)^{\operatorname{codim}\left(\mathcal{M}^{\lambda}\right)} \chi^{\operatorname{vir}}\left(\mathcal{M}^{\lambda}, V \otimes \mathrm{S}\left(T_{\mathcal{M}^{\lambda}}(\overline{\mathcal{M}})\right) \otimes \operatorname{det}\left(T_{\mathcal{M}^{\lambda}} \overline{\mathcal{M}}\right)\right)^{G} .
$$

Now the Thom isomorphism 5.2 (a) 1 and isomorphism $\mathcal{M}^{\lambda} \cong G \times_{R_{\lambda}} \mathcal{Y}^{\lambda}$ imply

$$
\begin{aligned}
& \chi^{\operatorname{vir}}\left(\mathcal{M}^{\lambda}, V \otimes \mathrm{S}\left(T_{\mathcal{M}^{\lambda}} \overline{\mathcal{M}}\right) \otimes \operatorname{det}\left(T_{\mathcal{M}^{\lambda}} \overline{\mathcal{M}}\right)\right)^{G} \\
= & \chi^{\operatorname{vir}}\left(\mathcal{Y}^{\lambda, s s}, V \otimes \mathrm{S}\left(T_{\mathcal{M}^{\lambda}} \overline{\mathcal{M}}\right) \otimes \operatorname{det}\left(T_{\mathcal{M}^{\lambda}} \overline{\mathcal{M}}\right) \otimes \underline{\Lambda}\left(\mathfrak{g} / \mathfrak{r}_{\lambda}\right)\right)^{G_{\lambda}} \\
= & \chi^{\operatorname{vir}}\left(\mathcal{Z}^{\lambda, s s}, V \otimes S\left(T_{\mathcal{M}^{\lambda}} \overline{\mathcal{M}} \oplus T_{\mathcal{Z}^{\lambda}}^{\vee} \mathcal{Y}^{\lambda}\right) \otimes \operatorname{det}\left(T_{\mathcal{M}^{\lambda}} \overline{\mathcal{M}}\right) \otimes \underline{\Lambda}\left(\mathfrak{g} / \mathfrak{r}_{\lambda}\right)\right)^{G_{\lambda}} .
\end{aligned}
$$

We apply the formula in a situation where the only contribution comes from the open stratum. Let $\mathcal{O}_{X}(1)$ be the hyperplane bundle for $X$. We construct a suitable determinant line bundle on $\overline{\mathcal{M}}_{n}^{G}\left(\mathbb{P}^{1}, X\right)_{0}$ as follows. Let $p: \overline{\mathcal{U}}_{n}^{G}\left(\mathbb{P}^{1}, X\right)_{0} \rightarrow$ $\overline{\mathcal{M}}_{n}^{G}\left(\mathbb{P}^{1}, X\right)_{0}$ denote the universal curve, and $q: \overline{\mathcal{U}}_{n}^{G}\left(\mathbb{P}^{1}, X\right)_{0} \rightarrow \mathbb{P}^{1}$ the morphism given by $(C, \pi: P \rightarrow \Sigma, u: C \rightarrow P(X), \underline{z}, w \in C) \mapsto \pi(u(w))$. Define

$$
D(k)=\operatorname{det}\left(R p_{*} e^{*} \mathcal{O}_{X}(1) \otimes q^{*} \mathcal{O}_{\mathbb{P}^{1}}(k)\right) ;
$$

the effect of the twist by $\mathcal{O}_{\mathbb{P}^{1}}(k)$ is to make the contribution from the principal component dominant.

Lemma 5.6. For any $\lambda$ and class $d \in H_{2}(X)$, there exists a constant $c$ such that the weight of $\mathbb{C}_{\lambda}^{*}$ on $D(k) \mid \mathcal{Z}^{\lambda}$ is at least $k(\lambda, \lambda)-c$.

Proof. We can compute the weight by Riemann-Roch applied to any $\mathbb{P}^{1}$-fiber of $p$ over $\mathcal{Z}^{\lambda}$. For any $\mathbb{C}_{\lambda}^{*}$-equivariant line bundle $L$ over $\overline{\mathcal{U}}_{n}^{G}\left(\mathbb{P}^{1}, X\right)_{0} \mid \mathcal{Z}^{\lambda}$ we write the $\mathbb{C}_{\lambda}^{*}$-equivariant Chern class of $L$ as a sum $c_{1}(L)+\mu_{L, \lambda}$, where $\mu_{L, \lambda}$ is the weight on $L$. The weight of $\mathbb{C}_{\lambda}^{*}$ acting on the push-forward $\operatorname{det}\left(R p_{*} e^{*} \mathcal{O}_{X}(1) \otimes q^{*} \mathcal{O}_{\mathbb{P}^{1}}(k)\right)$ is

\footnotetext{
${ }^{1}$ Erratum added after publication: The proof of Theorem 5.5 is not quite correct because for the derived structures on the strata as we have defined them the assumptions in 5.2 (b) may not hold. A more general non-abelian localization theorem, which applies to the case at hand by [10, Appendix], has been proved by Daniel Halpern-Leistner [11, (5)].
} 
the equivariant part of the equivariant first Chern class of the push-forward of the Chern character of $e^{*} \mathcal{O}_{X}(1) \otimes q^{*} \mathcal{O}_{\mathbb{P}^{1}}(k)$. The dominant contribution, linear in $k$, is

$$
\left(\int_{\mathbb{P}^{1}} c_{1}\left(\mathcal{O}_{\mathbb{P}^{1}}(k)\right)\right) \mu_{\mathcal{O}_{X}(1), \lambda}=k(\lambda, \lambda) .
$$

The actual weight is given by the above formula plus a zeroth order term, as claimed.

We denote by $\kappa_{G}(V)$ resp. $\kappa_{T}(V)$ the quotient of a $G$-equivariant vector bundle $V$ on $\overline{\mathcal{M}}_{0, n}\left(\mathbb{P}^{1} \times X,(1, d)\right)$ to $\overline{\mathcal{M}}_{n}^{G}\left(\mathbb{P}^{1}, X,(1, d)\right)_{0}$ resp. $\overline{\mathcal{M}}_{n}^{T}\left(\mathbb{P}^{1}, X,(1, d)\right)_{0}$.

Corollary 5.7. For any $G$-vector bundle $V$ over $\overline{\mathcal{M}}_{0, n}\left(\mathbb{P}^{1} \times X,(1, d)\right)$, for $k \gg 0$ we have

$$
\chi^{\mathrm{vir}}\left(\overline{\mathcal{M}}_{n}^{G}\left(\mathbb{P}^{1}, X, d\right)_{0}, \kappa_{G}(V \otimes D(k))\right)=\chi^{\mathrm{vir}}\left(\overline{\mathcal{M}}_{0, n}\left(\mathbb{P}^{1} \times X,(1, d)\right), V \otimes D(k)\right)^{G} .
$$

Proof. By Theorem 5.5 and Lemma 5.6, for $k \gg 0$ the only contribution to the invariant part $\chi^{\operatorname{vir}}\left(\overline{\mathcal{M}}_{0, n}\left(\mathbb{P}^{1}, X, d\right), \kappa_{G}(V)\right)^{G}$ comes from the 0 -semistable stratum. The result now follows from Proposition 5.1 .

The final step is pass to cohomology using the virtual Riemann-Roch formula proved in Tonita [38]. To justify the application of the virtual Riemann-Roch formula, we must show that $\overline{\mathcal{M}}_{n}^{G}\left(\mathbb{P}^{1}, X, d\right)_{0}$ embeds in a non-singular proper DeligneMumford stack. For this note that $\overline{\mathcal{M}}_{0, n}\left(\mathbb{P}^{1} \times X,(1, d)\right)$ embeds equivariantly in some $\overline{\mathcal{M}}_{0, n}\left(\mathbb{P}^{N}, \iota_{*}(1, d)\right)$ which is non-singular, where $\iota: \mathbb{P}^{1} \times X \rightarrow \mathbb{P}^{N}$ is a projective embedding, and so $\overline{\mathcal{M}}_{n}^{G}\left(\mathbb{P}^{1}, X, d\right)_{0}$ embeds in $\overline{\mathcal{M}}_{0, n}\left(\mathbb{P}^{N}, \iota_{*}(1, d)\right) / / G$. Now as in Kirwan 23] we may assume, after blowing up recursively the orbit-type strata, that $\overline{\mathcal{M}}_{0, n}\left(\mathbb{P}^{N}, \iota_{*}(1, d)\right) / / G$ is a locally free quotient and so a non-singular proper Deligne-Mumford stack.

Corollary 5.8. Suppose that every 0-semistable gauged map has finite automorphism group. For any $G$-equivariant algebraic vector bundle $V$ on $\overline{\mathcal{M}}_{0, n}\left(\mathbb{P}^{1} \times X,(1, d)\right)$,

$$
\chi^{\operatorname{vir}}\left(\overline{\mathcal{M}}_{n}^{G}\left(\mathbb{P}^{1}, X, d\right)_{0}, \kappa_{G}(V)\right)=(\# W)^{-1} \chi^{\operatorname{vir}}\left(\overline{\mathcal{M}}_{n}^{T}\left(\mathbb{P}^{1}, X, d\right)_{0}, \kappa_{T}(V \otimes \underline{\Lambda}(\mathfrak{g} / \mathfrak{t}))\right) .
$$

Proof. By Corollary [5.7, the statement of Corollary 5.8 holds for bundles of the form $V \otimes D(k)$ for $k \gg 0$. Virtual Riemann-Roch implies that the virtual Euler characteristics $\chi^{\operatorname{vir}}\left(\overline{\mathcal{M}}_{n}^{G}\left(\mathbb{P}^{1}, X, d\right)_{0}, \kappa_{G}(V \otimes D(k))\right)$ and $\chi^{\operatorname{vir}}\left(\overline{\mathcal{M}}_{n}^{T}\left(\mathbb{P}^{1}, X, d\right)_{0}, \kappa_{T}(V \otimes\right.$ $D(k) \otimes \underline{\Lambda}(\mathfrak{g} / \mathfrak{t})))$ are quasipolynomial in $k$, and the claim follows.

Taking highest order terms on both sides of Corollary 5.8 under the Adams operations, as in Teleman-Woodward [37, Section 5], gives

Corollary 5.9. Suppose that any 0-semistable gauged map has finite automorphism group. Then for any $d \in H_{2}^{G}(X, \mathbb{Z})$ and $G$-equivariant algebraic vector bundle $V$ on 


$$
\begin{aligned}
& \left.\overline{\mathcal{M}}_{0, n}\left(\mathbb{P}^{1} \times X,(1, d)\right)\right) \\
& \int_{\left[\overline{\mathcal{M}}_{n}^{G}\left(\mathbb{P}^{1}, X, d\right)_{0}\right]} \kappa_{G}\left(\mathrm{Ch}_{G}(V)\right)=(\# W)^{-1} \int_{\left[\overline{\mathcal{M}}_{n}^{T}\left(\mathbb{P}^{1}, X, d\right)_{0}\right]} \kappa_{T}\left(\mathrm{Ch}_{G}(V) \cup \operatorname{Eul}(\operatorname{Ind}(\mathfrak{g} / \mathfrak{t}))\right) .
\end{aligned}
$$

Combining with the main result Theorem 1.2 gives the abelianization Theorem 1.3 for genus zero gauged Gromov-Witten invariants in the "small area" chamber, for classes of the form $\alpha=\mathrm{Ch}_{G}(V)$ for algebraic vector bundles $V$. By FultonMacPherson 9, any class on $\bar{M}_{n}\left(\mathbb{P}^{1}\right)$ is of this type. By combining these results with those of [14], one obtains the same result for arbitrary $\rho$ under suitable finite automorphism assumptions.

\section{Construction of a cobordism}

One expects the equality of the zero-area gauged Gromov-Witten invariants with the small-area gauged Gromov-Witten invariants in Theorem 1.2 to hold more generally for any Hamiltonian $K$-manifold. We prove the equality in the case that every vortex is regular, by constructing a cobordism between the two moduli spaces, namely a differentiable structure on the union

$$
\bar{M}_{n}^{K}\left(\mathbb{P}^{1}, X\right)_{[0, \rho]}:=\bigcup_{\rho^{\prime} \in[0, \rho]} \bar{M}_{n}^{K}\left(\mathbb{P}^{1}, X\right)_{\rho^{\prime}} .
$$

Let $\bar{M}^{K}\left(\mathbb{P}^{1}, X\right)_{(0, \rho]}$ denote the locus with non-zero vortex parameter. These spaces have natural topologies defined by Gromov convergence of the sections and $C^{0}$ convergence of the connections, up to gauge transformation. The main result of this section is:

Theorem 6.1. Suppose that every element of $\bar{M}_{n}^{K}\left(\mathbb{P}^{1}, X\right)_{0}$ is regular. For $\rho$ sufficiently small, $\bar{M}_{n}^{K}\left(\mathbb{P}^{1}, X\right)_{[0, \rho]}$ has the structure of an oriented stratified-smooth orbifold, giving an oriented stratified-smooth orbifold cobordism between $\bar{M}_{n}^{K}\left(\mathbb{P}^{1}, X\right)_{\rho}$ and $\bar{M}_{n}^{K}\left(\mathbb{P}^{1}, X\right)_{0}$.

6.1. Approximation for zero-area vortices. First we show that a regular zeroarea vortex may be approximated by sequences of small-area vortices. Let $A$ be the trivial connection on $\Sigma \times K$.

Theorem 6.2. Given a regular zero-area-vortex $u:=\left(u_{0}, \ldots, u_{m}\right)$ of combinatorial type $\Gamma$ there exists a neighborhood $U$ of 0 in $\operatorname{Def}_{\Gamma}(u)$ and constants $c_{0}, \rho_{0}>0$ such that for $\rho<\rho_{0}$ and $(a, v) \in U$ there exists a unique $\left(a_{\rho}, v_{\rho}\right)$ depending smoothly on $a, v$ such that $\left(A+\pi^{*} a_{\rho}, u_{\rho}=\exp _{u}\left(v+v_{\rho}\right)\right)$ is a $\rho$-vortex of combinatorial type $\Gamma$ in Coulomb gauge with respect to $(A, u),\left\|\left(a_{\rho}, v_{\rho}\right)\right\|<c_{0} \rho^{3 / 2}$, and $\left(a_{\rho}, v_{\rho}\right)$ in the image of the right inverse $Q^{0, \rho}$ of Lemma 6.4.

The strategy of proof is to construct approximate solution and then use an iteration to construct an exact solution. It has in common with the analogous theorem in 
Gaio-Salamon 12] for the large area limit that the quadratic term does not satisfy a uniform bound. However, our case is easier because the space on which the quadratic term is not uniformly bounded is finite dimensional. The approximation theorem implies that $\bar{M}^{K}\left(\mathbb{P}^{1}, X\right)_{0}$ is contained in the closure of the union of moduli spaces $\bar{M}^{K}\left(\mathbb{P}^{1}, X\right)_{\rho}, \rho \leq \rho_{0}$, so that it provides a compactification in the usual sense. Given an zero-area-vortex $(A, u)$ with $A$ the trivial connection we consider the equation

$$
F_{A+\pi^{*} a}+\rho \operatorname{Vol}_{\mathbb{P}^{1}}\left(\exp _{u} v\right)^{*} \Phi=0 .
$$

Consider the Hodge splitting

$$
\Omega^{2}\left(\mathbb{P}^{1}, \mathfrak{k}\right)_{1, p}=\operatorname{Ker}\left(\mathrm{d}^{*}\right) \oplus \operatorname{Im}(\mathrm{d}) \cong \mathfrak{k} \oplus \operatorname{Im}(\mathrm{d}) .
$$

We denote by $\pi_{0}$ resp. $\pi_{1}$ the projection on the first resp. second factor,

$$
\pi_{0}(v)=\left(\int_{\mathbb{P}^{1}} v\right) \operatorname{Vol}_{\mathbb{P}^{1}}, \quad \pi_{1}(v)=v-\pi_{0}(v) .
$$

Consider the following modification of (6) by using Banach norms given by multiplying the standard $W^{1, p}$ resp. $L^{p}, p>2$ norms on the harmonic pieces by $\rho$.

$$
\begin{gathered}
\mathcal{F}_{A, u}^{0, \rho}: \Omega^{0}\left(\mathbb{P}^{1}, \mathfrak{k}\right)_{1, p} \oplus \Omega^{0}\left(\mathbb{P}^{1}, u^{*} T X\right)_{1, p} \rightarrow \operatorname{Im}\left(\mathrm{d}_{A} \oplus \mathrm{d}_{A} *\right)_{0, p} \oplus(\mathfrak{k} \oplus \mathfrak{k}) \oplus \Omega^{0,1}\left(\mathbb{P}^{1}, u^{*} T X\right)_{0, p} \\
\mathcal{F}_{A, u}^{0, \rho}:\left[\begin{array}{c}
a \\
v
\end{array}\right] \mapsto\left[\begin{array}{c}
\phi \\
\psi \\
\mu \\
\lambda \\
\eta
\end{array}\right]:=\left[\begin{array}{l}
\pi_{1}\left(F_{A+\pi^{*} a}+* \rho \exp _{u}(v)^{*} \Phi\right) \\
\pi_{1}\left(\mathrm{~d}_{A} * a\right) \\
\pi_{0}\left(* \exp _{u}(v)^{*} \Phi+\rho^{-1}([a, a] / 2)\right) \\
\pi_{0} * L_{J v} \Phi \\
\Psi_{u}(v)^{-1} \bar{\partial}_{A+\pi^{*} a} \exp _{u}(v)
\end{array}\right] .
\end{gathered}
$$

Here we have used that the term $\int_{\mathbb{P 1}} \mathrm{d}_{A} a$ vanishes by Stokes' theorem. The map $\mathcal{F}_{A, u}^{0, \rho}$ of (19) is a smooth map of Banach spaces, by standard Sobolev multiplication theorems. The double subscript in $\mathcal{F}_{A, u}^{0, \rho}$ is meant to indicate that we view $\mathcal{F}_{A, u}^{\rho}$ as a map with respect to the Banach spaces for zero area introduced above.

Definition 6.3. Let $\tilde{D}_{a, v}^{0, \rho}$ denote the linearization of $\mathcal{F}_{A, u}^{0, \rho}$ at $(a, v)$, that is,

$$
\tilde{D}_{a, v}^{0, \rho}\left(a_{1}, v_{1}\right)=\left[\begin{array}{l}
\pi_{1}\left(\mathrm{~d}_{A+\pi^{*} a} a_{1}+\rho * L_{v_{1}} \Phi\right) \\
\pi_{1}\left(\mathrm{~d}_{A} * a_{1}\right) \\
\pi_{0}\left(* L_{v_{1}} \Phi+\rho^{-1}\left[a, a_{1}\right] / 2\right) \\
\pi_{0} * L_{J v_{1}} \Phi \\
D_{A+\pi^{*} a, \exp _{u}(v)}\left(a_{1}, v_{1}\right)
\end{array}\right] .
$$

The operators $\tilde{D}_{0, v}^{0, \rho}$ (that is, with $a=0$ ) have limit $\tilde{D}_{0, v}^{0,0}$ given by

$$
\tilde{D}_{0, v}^{0,0}\left(a_{1}, v_{1}\right)=\left[\begin{array}{l}
\pi_{1}\left(\mathrm{~d}_{A} a_{1}\right) \\
\pi_{1}\left(\mathrm{~d}_{A} * a_{1}\right) \\
\pi_{0} * L_{v_{1}} \Phi \\
\pi_{0} * L_{J v_{1}} \Phi \\
D_{A, \exp _{u}(v)}\left(a_{1}, v_{1}\right)
\end{array}\right]
$$


We wish to compare this operator with the linearized operator $\tilde{D}_{u}^{0}$ for the zeroarea vortex $u$ of (12), which does not have a gauge-theoretic part. Let $A$ be the trivial connection. Note that a zero-area vortex $u$ is regular iff $\tilde{D}_{0,0}^{0, \rho}$ is surjective for $\rho$ sufficiently small. Indeed, since the operator $\mathrm{d}_{A} \oplus \mathrm{d}_{A}^{*}$ for the trivial connection $A$ is surjective onto the first component in the Hodge decomposition (18), $\tilde{D}_{0,0}^{0,0}$ is surjective iff $\tilde{D}_{u}^{0}$ is.

Next we introduce suitable right inverses for the operators $\tilde{D}_{0,0}^{0, \rho}$.

Lemma 6.4. If $(A, u)$ is a regular zero-area-vortex then the linearization $\tilde{D}_{0,0}^{0,0}$ has a uniformly bounded right inverse $Q^{0,0}$ with the following property: There exists a constant $c>0$ such that if $Q^{0,0}(\phi, \psi, \mu, \lambda, \eta)=(a, v)$ then

$$
\|a\| \leq c\|\phi, \psi\|, \quad\|v\| \leq c\|\phi, \psi, \mu, \lambda, \eta\| .
$$

Proof. Define $Q^{0,0}(\phi, \psi, \mu, \lambda, \eta)=(a, v)$ where $a=\left(\mathrm{d}_{A} \oplus \mathrm{d}_{A}^{*}\right)^{-1}(\phi, \psi)$, the right inverse to $\mathrm{d}_{A} \oplus \mathrm{d}_{A}^{*}$ is given by the Hodge splitting, and

$$
\left(\pi_{0} L_{v} \Phi, \pi_{0} L_{J v} \Phi, D_{A, u}(0, v)\right)=\left(\mu, \lambda, \eta-D_{A, u}(a, 0)\right)
$$

The claimed properties follow from standard Sobolev multiplication theorems.

Corollary 6.5. If $(A, u)$ is a regular zero-area-vortex then the linearization $\tilde{D}_{0,0}^{0, \rho}$ has a uniformly bounded right inverse $Q^{0, \rho}$ with $Q^{0, \rho}=Q_{0}^{0, \rho}+\rho Q_{1}^{0, \rho}$ for some uniformly bounded operator $Q_{1}^{0, \rho}$, and $Q_{0}^{0, \rho}(\phi, \psi, \mu, \lambda, \eta)=(a, v)$ with $\|a\| \leq c\|\phi, \psi\|$ and $\|v\| \leq$ $c\|\phi, \psi, \mu, \lambda, \eta\|$.

Proof. Since $\tilde{D}_{0,0}^{0, \rho}=\tilde{D}_{0,0}^{0,0}+\rho \tilde{D}_{0,0}^{0, \rho,{ }^{\prime}}$ where $\tilde{D}_{0}^{0, \rho,{ }^{\prime}}\left(a_{1}, v_{1}\right)=\left(\pi_{1}\left(* L_{v_{1}} \Phi\right), 0,0,0,0\right)$.

Lemma 6.6. The map $\mathcal{F}_{A, u}^{0, \rho}$ satisfies a uniform quadratic estimate except for a term quadratic in a which has norm linear in $\rho^{-1}$ :

$$
\mathcal{F}_{A, u}^{0, \rho}\left(a+a_{1}, v+v_{1}\right)-\mathcal{F}_{A, u}^{0, \rho}(a, v)=\tilde{D}_{a, v}^{0, \rho}\left(a_{1}, v_{1}\right)+(\phi, 0, \mu, 0, \eta)
$$

where

$$
\|\phi\| \leq c_{1}\left(\left\|a_{1}\right\|^{2}+\rho\left\|v_{1}\right\|^{2}\right) \quad\|\mu\| \leq c_{2}\left(\left\|v_{1}\right\|^{2}+\rho^{-1}\left\|a_{1}\right\|^{2}\right) \quad\|\eta\| \leq c_{3}\left\|a_{1}, v_{1}\right\|^{2}
$$

for some constants $c_{1}, c_{2}, c_{3}$ depending on $\|A\|,\|u\|$ and a bound on $\|a\|,\|v\|$. 
Proof. Define $\phi, \mu, \eta$ by

$$
\begin{gathered}
\mathcal{F}_{A, u}^{0, \rho}\left(a+a_{1}, v+v_{1}\right) \\
-\mathcal{F}_{A, u}^{0, \rho}(a, v)
\end{gathered}=\left[\begin{array}{c}
\pi_{1}\left(\begin{array}{c}
\mathrm{d}_{A+\pi^{*} a} a_{1}+\left[a_{1}, a_{1}\right] / 2+\rho \mathrm{Vol}_{\mathbb{P} 1} \\
\left(* \exp _{u}\left(v+v_{1}\right)^{*} \Phi-\exp _{u}(v)^{*} \Phi\right)
\end{array}\right) \\
\pi_{1}\left(\mathrm{~d}_{A} * a_{1}\right) \\
\pi_{0}\left(\begin{array}{c}
* \exp _{u}\left(v+v_{1}\right)^{*} \Phi-\exp _{u}(v)^{*} \Phi \\
+\rho^{-1}\left(\mathrm{~d}_{A+\pi^{*} a} a_{1}+\left[a_{1}, a_{1}\right] / 2\right)
\end{array}\right) \\
\pi_{0}\left(* L_{J v_{1}} \Phi\right) \\
\Psi_{u}\left(v+v_{1}\right)^{-1} \bar{\partial}_{A+\pi^{*}\left(a+a_{1}\right)} \exp _{u}\left(v+v_{1}\right)- \\
\Psi_{u}(v)^{-1} \bar{\partial}_{A+a} \exp _{u}(v)
\end{array}\right]
$$

The claimed estimates follow.

Proof of Theorem 6.2. We give the proof in the case that $(A, u)$ has no bubbles; the general case is similar and left to the reader. We use Newton iteration to find $a_{\rho}, v_{\rho}$ such that $\mathcal{F}_{A, u}^{0, \rho}\left(a_{\rho}, v_{\rho}\right)=0$. To solve the Newton iteration we must show that our initial condition is a sufficiently approximate solution so that the blow-up of the quadratic term does not affect convergence of the iteration. Let $Q^{0, \rho}$ denote the right inverse of Lemma 6.4. We define by induction a sequence

$$
\hat{\zeta}_{\nu}=-Q^{0, \rho} \mathcal{F}_{A, u}^{0, \rho} \zeta_{\nu}, \quad \zeta_{\nu+1}=\zeta_{\nu}+\hat{\zeta}_{\nu}
$$

such that

$$
\hat{\zeta}_{\nu}=\left(\hat{a}_{\nu}, \hat{v}_{\nu}\right), \quad\left\|\hat{a}_{\nu}\right\| \leq c_{0} \rho^{\nu}, \quad\left\|\hat{v}_{\nu}\right\| \leq c_{0} \rho^{\nu-1} .
$$

To get the iteration started we define

$$
\zeta_{1}=\hat{\zeta}_{0}=-Q^{0, \rho}\left(\rho * \exp _{u}(v)^{*} \Phi, 0,0,0,0\right) .
$$

Hence $\zeta_{1}=\left(a_{1}, v_{1}\right)$ and $\left\|\zeta_{1}\right\| \leq c_{4} \rho$ with $c_{4}$ depending on $\sup _{x \in X}|\Phi(x)|$ and the norm of $Q^{0, \rho}$ which is uniformly bounded by Lemma 6.4. Define

$$
\hat{\zeta_{1}}=-Q^{0, \rho} \mathcal{F}^{0, \rho}\left(\zeta_{1}\right), \quad \zeta_{2}=\zeta_{1}+\hat{\zeta}_{1}=\left(a_{2}, v_{2}\right) .
$$

Then

$$
\mathcal{F}_{A, u}^{0, \rho}\left(\zeta_{2}\right)=\left(\phi_{2}, 0, \mu_{2}, 0, \eta_{2}\right)
$$

and by Lemma 6.6

$$
\left\|\phi_{2}\right\|,\left\|\eta_{2}\right\|<c_{5} \rho^{2}, \quad\left\|\mu_{2}\right\|<c_{6} \rho
$$

for some constants $c_{5}, c_{6}$ depending on $c_{1}, c_{2}, c_{3}$. Using Lemma 6.4 we have

$$
\hat{\zeta}_{2}=\left(\hat{a}_{2}, \hat{v}_{2}\right), \quad\left\|\hat{a}_{2}\right\|<c_{7} \rho^{2}, \quad\left\|\hat{v}_{2}\right\|<c_{8} \rho
$$

for some constants $c_{7}, c_{8}$ depending on the previous constants and the norm of $Q_{1}$.

Suppose that the sequence $\hat{\zeta}_{1}, \ldots, \hat{\zeta}_{\nu-1}$ constructed in this way satisfies the hypotheses (23). By Lemma 6.6, there exists a constant $c_{9}>0$ depending on the previous constants such that

$$
\left(\phi_{\nu}, \psi_{\nu}, \mu_{\nu}, \lambda_{\nu}, \eta_{\nu}\right):=\mathcal{F}_{A, u}^{0, \rho}\left(\zeta_{\nu}\right)
$$


satisfies for $\rho$ such that for $\rho<3 c_{0} c_{9}$

$$
\begin{aligned}
\left\|\phi_{\nu}\right\| & <c_{9}\left(\left\|a_{\nu}\right\|^{2}+\rho\left\|v_{\nu}\right\|^{2}\right)<c_{9}\left(c_{0}^{2} \rho^{2 \nu}+c_{0}^{2} \rho^{2(\nu-1)+1}\right)<c_{0} \rho^{\nu+1} \\
\left\|\mu_{\nu}\right\| & <c_{9}\left(\left\|v_{\nu}\right\|^{2}+\rho^{-1}\left\|a_{\nu}\right\|^{2}\right)<c_{9} c_{0}^{2}\left(\rho^{2(\nu-1)}+\rho^{2 \nu-1}\right)<c_{0} \rho^{\nu} \\
\left\|\eta_{\nu}\right\| & <c_{9}\left\|a_{1}, v_{1}\right\|^{2}<c_{9} c_{0}^{2}\left(\rho^{2 \nu}+\rho^{2 \nu-1}+\rho\right)^{2(\nu-1)}<c_{0} \rho^{\nu} .
\end{aligned}
$$

Applying $Q^{0, \rho}$ gives an element $\hat{\zeta}_{\nu}=\left(\hat{a}_{\nu}, \hat{v}_{\nu}\right)$ satisfying (23) , as required. Because $\left\|\hat{\zeta}_{\nu}\right\|<c_{0} \rho^{\nu}, \zeta_{\nu}$ is a Cauchy sequence and converges to a limit $\zeta_{\rho}=\left(a_{\rho}, v_{\rho}\right)$ with

$$
\left\|\zeta_{\rho}-\zeta_{\nu}\right\|<c_{0} \rho^{\nu+1} /(1-\rho)<c_{0} \rho^{\nu+1} .
$$

To prove uniqueness, suppose that $\zeta_{\rho}^{\prime}=\left(a_{\rho}^{\prime}, v_{\rho}^{\prime}\right)$ is another solution with

$$
\left\|\zeta_{\rho}-\zeta_{\rho}^{\prime}\right\|<c_{0} \rho^{3 / 2}
$$

Then

$$
0=\mathcal{F}_{A, u}^{0, \rho}\left(a_{\rho}, v_{\rho}\right)-\mathcal{F}_{A, u}^{0, \rho}\left(a_{\rho}^{\prime}, v_{\rho}^{\prime}\right)=D_{0}^{\rho, 0}\left(a_{\rho}-a_{\rho}^{\prime}, v_{\rho}-v_{\rho}^{\prime}\right)+\epsilon
$$

for some $\epsilon$. By the uniform quadratic estimate in Lemma 6.6 and (25)

$$
\|\epsilon\|<c_{0} \rho .
$$

Since $\left(a_{\rho}-a_{\rho}^{\prime}, v_{\rho}-v_{\rho}^{\prime}\right)$ lies in the image of $Q^{0, \rho}$, we have

$$
\left\|a_{\rho}-a_{\rho}^{\prime}, v_{\rho}-v_{\rho}^{\prime}\right\| \geq c
$$

which contradicts (26) for $c_{0}$ sufficiently small. Smooth dependence follows from the implicit function theorem.

The approximation theorem 6.2 extends to the case with bubbles as follows. For any collection of gluing parameters $\underline{\delta}=\left(\delta_{1}, \ldots, \delta_{m}\right)$ and $v \in \Omega^{0}\left(C, u^{*} T X\right)$, let $\exp _{u \underline{\delta}}\left(v^{\underline{\delta}}\right): C^{\underline{\delta}} \rightarrow X$ the glued zero-area vortex constructed using the implicit function theorem as in [27, Section 10], so that

$$
\operatorname{Def}(u) \rightarrow \bar{M}^{K}\left(\mathbb{P}^{1}, X\right)_{0}, \quad(v, \underline{\delta}) \mapsto \exp _{u \underline{\underline{\delta}}}\left(v^{\underline{\delta}}\right)
$$

gives local charts for $\bar{M}^{K}\left(\mathbb{P}^{1}, X\right)_{0}$ as in [15]. Thus $C$ is obtained from $C$ by removing small balls around the nodes, and gluing together the components using maps $\kappa_{j}^{+}=$ $\kappa_{j}^{-} / \delta_{j}$, where $\kappa_{j}^{ \pm}$are fixed local coordinates around the nodes, $u \underline{\underline{\delta}}$ is an approximate solution constructed using cutoff functions, and $v^{\underline{\delta}}$ is the correction provided by the implicit function theorem. Given a set of markings $\underline{z}$ on $C$ in the complement of the domain of the local coordinates near the nodes we denote by $\underline{z}^{\underline{\delta}}$ the markings on $C \underline{\underline{\delta}}$. We now wish to combine the gluing construction for pseudoholomorphic maps with the approximation Theorem 6.2. This will give rise to charts for our moduli space near $\rho=0$.

Theorem 6.7. Given a regular zero-area-vortex $u:=\left(u_{0}, \ldots, u_{m}\right)$ of combinatorial type $\Gamma$ there exists a neighborhood $U$ of 0 in $\operatorname{Def}(u)$ and constants $c_{0}, \rho_{0}>0$ such that for $\rho<\rho_{0}$ and $(a, v, \underline{\delta}) \in U$ there exists a unique $\left(a_{\rho}, v_{\rho}\right)$ such that

$$
\left(A_{\text {triv }}+a+a_{\rho}, u_{\rho}=\exp _{u \underline{\underline{\delta}}}\left(v^{\underline{\delta}}+v_{\rho}\right)\right)
$$


is a $\rho$-vortex in Coulomb gauge with respect to the trivial connection $A_{\text {triv }},\left\|a_{\rho}, v_{\rho}\right\|<$ $c_{0} \rho^{3 / 2}$, and $\left(a_{\rho}, v_{\rho}\right)$ in the image of the right inverse $Q^{0, \rho}$ of Lemma 6.4. Furthermore, $\left(a_{\rho}, v_{\rho}\right)$ depends stratified-smoothly on $v, \underline{\delta}$, that is, smoothly on the subset where the gluing parameters $\delta_{i}, i \in I$ are non-zero, for each $I \subset\{1, \ldots, m\}$.

Proof. Let $\exp _{u^{\underline{\delta}}}\left(v^{\underline{\delta}}\right)$ denote the zero-area vortex given by gluing. We wish to solve $\mathcal{F}_{A, \exp _{u} \underline{\underline{\delta}}(v \underline{\underline{\delta}})}^{0, \rho}\left(a+a_{\rho}, v+v_{\rho}\right)=0$ for $a_{\rho}, v_{\rho}$, using Newton iteration. The error term $\rho \exp _{u \underline{\underline{\delta}}}\left(v^{\underline{\delta}}\right)^{*} P(\Phi)$ is uniformly bounded in $\underline{\delta}$ and $\rho$, by the bound on $\Phi$. The norms of the operators $Q^{0, \rho}$ and $\tilde{D}^{0, \rho}$ at $\exp _{u \underline{\underline{\delta}}}\left(v^{\underline{\delta}}\right)$ are uniformly bounded in $\underline{\delta}$ and $\rho$, that is, $\left\|Q^{0, \rho}\right\|<c$ and $\left\|\tilde{D}^{0, \rho}\right\|<c$ for some $\rho$-independent constant $c$. Indeed, the inverse to $D_{\exp _{u} \underline{\delta}(v \underline{\delta})}$ is uniformly bounded in $\underline{\delta}$, by the gluing argument for pseudoholomorphic maps in [27, Chapter 10]. The claim now follows from Lemma 6.4. The map $\mathcal{F}_{A, u, \underline{\delta}}^{0, \rho}$ satisfies a quadratic estimate uniformly in $\underline{\delta}$ and uniformly in $\rho$ except for a term quadratic in $a$ which has norm linear in $\rho$, just as in Lemma 6.6. The same argument as in the case of smooth domain in Theorem 6.2 gives a solution $a_{\rho}, v_{\rho}$ by Newton iteration. Smoothness on each stratum follows from smoothness of $v^{\underline{\delta}}$ on $v, \underline{\delta}$ and the smoothness statement of Theorem 6.2.

6.2. Surjectivity. Let $u$ be a stable zero-area vortex. Let $\operatorname{Def}(u)_{\epsilon}$ denote a $\epsilon$-ball around 0 in the space $\operatorname{Def}(u)$ of infinitesimal deformations of (14). Theorem 6.2 defines for $\epsilon$ sufficiently small a map

$$
T_{u}^{\rho}: \operatorname{Def}(u)_{\epsilon} \rightarrow \bar{M}^{K}\left(\mathbb{P}^{1}, X\right)_{\rho} .
$$

The collection of images of the maps $T_{u}^{\rho}$ covers $\bar{M}^{K}\left(\mathbb{P}^{1}, X\right)_{\rho}$ for $\rho$ sufficiently small:

Theorem 6.8. Suppose that every zero-area nodal vortex is regular. For any constant $c>0$ and $d \in H_{2}^{G}(X, \mathbb{Z})$, there exists $\rho_{0}>0$ such that if $(A, u)$ is a $\rho$-vortex with $\rho<\rho_{0}$ and homology class $d$ then $(A, u)$ is in the image of $T_{u^{\prime}}^{\rho}$ for some nodal zero-area vortex $u^{\prime}$.

Proof. Suppose that the assertion in the statement does not hold, that is, for every $\rho$ there exists a $\rho$-vortex $\left(A_{\rho}, u_{\rho}\right)$ of class $d$ not in the image of any map $T_{u^{\prime}}^{\rho}$. By the compactness Theorem 1.1, after passing to a subsequence and gauge transformations $\left(A_{\rho}, u_{\rho}\right)$ converges to a zero-area-vortex $u_{0}$. First consider the case that $u_{0}$ has smooth domain $C \cong \mathbb{P}^{1}$, hence $u_{\rho}$ has smooth domain as well. We write

$$
A_{\rho}=a_{\rho}, \quad u_{\rho}=\exp _{u_{0}}\left(v_{\rho}\right)
$$

for some $a_{\rho}, v_{\rho}$. We may assume that $\left(A_{\rho}, u_{\rho}\right)$ is in Coulomb gauge, that is,

$$
\mathrm{d}^{*} a_{\rho}=0, \quad E_{u_{0}}^{*} v_{\rho}=0 .
$$

Define

$$
\left(a_{1}, v_{1}\right)=Q^{0,0}\left(\pi_{1} \rho * u_{\rho}^{*} \Phi, 0,0,0,0\right)
$$


We have an estimate $\left\|a_{1}, v_{1}\right\|<c_{1} \rho$ where $c_{1}$ depends on the bound on $\Phi$ and the norm of $Q^{0,0}$. Consider the map

$$
\begin{aligned}
\mathcal{F}^{0,0}: \Omega^{0}\left(\mathbb{P}^{1}, \mathfrak{k}\right)_{1, p} \oplus \Omega^{0}\left(C,\left(u_{0}\right)^{*} T X\right)_{1, p} & \rightarrow \operatorname{Im}\left(\mathrm{d} \oplus \mathrm{d}^{*}\right)_{0, p} \oplus(\mathfrak{k} \oplus \mathfrak{k}) \oplus \Omega^{0,1}\left(C,\left(u_{0}\right)^{*} T X\right)_{0, p} \\
(a, v) & \mapsto\left(\pi_{1} F_{a}, \pi_{1} d^{*} v, \int_{\mathbb{P}^{1}} * \exp _{u_{0}}(v)^{*} \Phi, E_{u_{0}}^{*} v, \Psi_{u_{0}}(v)^{-1} \bar{\partial} \exp _{u_{0}}(v)\right) .
\end{aligned}
$$

Using (29), (30), we have

$$
\begin{aligned}
\left\|\mathcal{F}^{0,0}\left(a_{\rho}-a_{1}, v_{\rho}-v_{1}\right)\right\|= & \left\|\left[\begin{array}{c}
\pi_{1} F_{a_{\rho}-a_{1}} \\
\pi_{1} d^{*}\left(a_{\rho}-a_{1}\right) \\
\int_{\mathbb{P}^{1}} * \exp _{u_{0}}\left(v_{\rho}-v_{1}\right)^{*} \Phi \\
E_{u_{0}}^{*} v_{\rho} \\
\Psi_{u_{0}}(v)^{-1} \bar{\partial} \exp _{u_{0}}(v)- \\
\Psi_{u_{0}}\left(v-v_{\rho}\right)^{-1} \bar{\partial} \exp _{u_{0}}\left(v-v_{\rho}\right)
\end{array}\right]\right\| \\
= & \left\|\left[\begin{array}{c}
\pi_{1}\left(F_{a_{\rho}}-\mathrm{d}_{A} a_{1}+\frac{1}{2}\left[a_{1}, a_{1}\right]\right) \\
0 \\
\int_{\mathbb{P}^{1}} * \exp _{u_{0}}\left(v_{\rho}-v_{1}\right)^{*} \Phi \\
0 \\
\Psi_{u}\left(v_{\rho}-v_{1}\right)^{-1} \bar{\partial} \exp _{u_{0}}\left(v_{\rho}-v_{1}\right)- \\
\Psi_{u_{0}}\left(v_{\rho}\right)^{-1} \bar{\partial} \exp _{u_{0}}\left(v_{\rho}\right)
\end{array}\right]\right\| \\
\leq c_{2} \quad \rho^{2} &
\end{aligned}
$$

where $c_{2}$ depends on $c_{1}$ and the constants in the Sobolev multiplication theorems. By the implicit function theorem for the map $\mathcal{F}^{0,0}$, there exists a zero-area vortex $u^{\prime}=\exp _{u_{0}}(v)$ within $c_{3} \rho^{2}$ of $\left(A_{\rho}-a_{1}, \exp _{u}\left(v_{\rho}-v_{1}\right)\right)$ in the sense that

$$
\left\|A_{\rho}-a_{1}\right\|^{2}+\left\|v-v_{\rho}+v_{1}\right\|^{2}<c_{3}^{2} \rho^{4} .
$$

Now consider the $\rho$-vortex $\left(A_{\rho}^{\prime}, u_{\rho}^{\prime}\right):=T_{\rho, u_{0}}(v)=:\left(a_{\rho}^{\prime}, \exp _{u^{\prime}}\left(v_{\rho}^{\prime}\right)\right)$. As in the proof of Theorem 6.7 this vortex lies within $c_{4} \rho^{2}$ of the first step in the Newton iteration, $\left(A_{\rho, 1}^{\prime}, u_{\rho, 1}^{\prime}\right)=\left(a_{\rho, 1}, \exp _{u^{\prime}}\left(v_{\rho, 1}\right)\right)$ where

$$
\left(a_{\rho, 1}, v_{\rho, 1}\right)=Q^{0,0}\left(\pi_{1} * \rho\left(u^{\prime}\right)^{*} \Phi, 0,0,0,0\right) .
$$

Now $\left(a_{\rho}, v_{\rho}\right)$ lies within $c_{1} \rho^{2}$ of $\left(a_{\rho, 1}, v_{\rho, 1}\right)$ by (30) and hence within $c_{5} \rho^{2}$ of $\left(a_{\rho}^{\prime}, v_{\rho}^{\prime}\right)$. For $\rho$ sufficiently small, $c_{5} \rho^{2}<c_{0} \rho^{3 / 2}$.

Next we show that we may assume that $\left(a_{\rho}^{\prime}, v_{\rho}^{\prime}\right)$ lies in the image of the right inverse $Q^{0, \rho}$ for the linearized operator $\tilde{D}^{0, \rho}$ of $u^{\prime}$. Given $(a, v)$ as the previous paragraph, we claim that

$$
(a, v)=\left(a_{0}, v_{0}\right)+\left(a_{1}, v_{1}\right), \quad\left(a_{0}, v_{0}\right) \in \operatorname{ker} \tilde{D}^{0, \rho}, \quad\left(a_{1}, v_{1}\right) \in \operatorname{Im} Q^{0, \rho}
$$

with norm $\left\|\left(a_{1}, v_{1}\right)\right\| \leq c\left\|\left(a_{1}, v_{1}\right)\right\|$. For any $c>0$ there exists $\rho_{0}$ such that for $\rho<\rho_{0}$, $\left\|\tilde{D}^{0, \rho}\left(a_{0}, v_{0}\right)\right\| \leq c\left\|\left(a_{0}, v_{0}\right)\right\|$ for any $\left(a_{0}, v_{0}\right) \in \operatorname{ker} \tilde{D}^{0,0}$. Thus the space ker $\tilde{D}^{0,0}$ is transverse to the image of $Q^{0, \rho}$, for $\rho$ sufficiently small: it meets $\operatorname{Im} Q^{0, \rho}$ trivially and projects isomorphically onto ker $\tilde{D}^{0, \rho}$. By the inverse function theorem, any nearby 
pair $(a, v)$ is of the form (33). By the uniqueness statement in Theorem 6.7, $\left(A_{\rho}, u_{\rho}\right)$ is in the image of the map $T_{u^{\prime}}^{\rho}$.

The case with bubbles is similar, using a map obtained by combining $\mathcal{F}^{0,0}$ with the usual Cauchy-Riemann equation on the bubbles. By iteration one constructs a zero-area vortex $u^{\prime}$ and a pair $\left(a^{\prime}, v^{\prime}\right)$ such that $T_{u^{\prime}}^{\rho}\left(a^{\prime}, v^{\prime}\right)$ is within $c_{0} \rho^{3 / 2}$ of $(A, u)$. Surjectivity follows from the uniqueness part of the implicit function theorem used in the gluing construction.

6.3. Construction of charts. The charts near zero area are given by the approximation construction of the previous section. Let $u$ be a zero-area vortex. Using (28) we have for $\rho$ sufficiently small a map

$$
T_{u}: \operatorname{Def}(u)_{\epsilon} \times[0, \rho] \rightarrow \bar{M}^{K}\left(\mathbb{P}^{1}, X\right)_{[0, \rho]} .
$$

Proposition 6.9. For $\epsilon, \rho$ sufficiently small $T_{u}$ is a homeomorphism onto an open neighborhood of $[u]$.

Sketch of proof. By Theorem 6.8, the image of $T_{u}$ contains an open neighborhood of $u$. We choose a complement of $\operatorname{aut}(C)$ in $\operatorname{ker} \tilde{D}_{u}$ and restrict $T_{u}$ to $v$ lying in this complement. Suppose that $\left(A, u^{\prime}\right)=T_{u}(v, \underline{\delta}, \rho)$. We claim that the derivative of $T_{u}$ at $(v, \underline{\delta}, \rho)$ is an injection for $(v, \underline{\delta}, \rho)$ sufficiently small. Indeed, the map $T_{u}$ is the composition of the gluing construction for pseudoholomorphic maps and the approximation construction for $\rho$-vortices given above. That the gluing construction has injective derivative is proved in [15], using the exponential gluing profile so that the evaluation maps at the additional markings are differentiable. It then suffices to consider the case without bubbles, for which we have

$$
0=\left.\frac{d}{d t}\right|_{t=0} \mathcal{F}^{\rho}\left(T_{u}^{\rho}\left(v+t v^{\prime}\right)\right)=\tilde{D}_{A, u^{\prime}}\left(D_{v} T_{u}^{\rho}\left(v^{\prime}\right)\right)=\tilde{D}_{A, u^{\prime}}\left(v^{\prime}+v^{\prime \prime}\right)
$$

for some $v^{\prime \prime}$ in the image of the right inverse $Q^{0, \rho}$. By Lemma 6.6, the image of the right inverse has trivial intersection with $\operatorname{ker}\left(\tilde{D}_{u}\right)$, which shows that $T_{u}$ is an injection. To show that $T_{u}$ is a homeomorphism, it remains to show that $T_{u}$ is open, that is, if $\left[A_{\nu}, u_{\nu}, \rho_{\nu}\right] \rightarrow\left[A, u^{\prime}, \rho\right]$ with $\left[A_{\nu}, u_{\nu}, \rho_{\nu}\right],\left[A, u^{\prime}, \rho\right]$ in the image then $v_{\nu} \rightarrow v$ and $\underline{\delta}_{\nu} \rightarrow \underline{\delta}$. The injectivity argument in [15] shows that $\underline{\delta}_{\nu} \rightarrow \underline{\delta}$. By Theorem 6.7 . $T_{u}\left(v_{\nu}, \underline{\delta}, \rho\right) \rightarrow T_{u}(v, \underline{\delta}, \rho)$ implies $v_{\nu} \rightarrow v$.

Proof of Theorem 6.1. That the moduli spaces $\bar{M}_{n}^{K}\left(\mathbb{P}^{1}, X\right)_{(0, \rho]}$ are stratified-smooth orbifolds for $\rho$ sufficiently small is [14, Theorem 6.2.3], using the fact that the $U(1)$ action on the moduli space of polarized vortices is free for $\rho$ sufficiently small. Charts for $\bar{M}_{n}^{K}\left(\mathbb{P}^{1}, X\right)_{[0, \rho]}$ near zero-area vortices are given by the maps $T_{u}$. By Proposition 6.9. $T_{u}$ is a homeomorphism onto an open neighborhood of $u$, and so defines a chart. Independence of the choice of complement of aut $(C)$ (given, for example, by choosing auxiliary hypersurfaces defining markings to make the domain curve stable) is similar to the case of pseudoholomorphic maps and left to the reader. An orientation for the moduli space is constructed from orientations on the determinant 
lines of the linearized operators $\tilde{D}_{A, u}$, induced from the deformation to the sum of $\mathrm{d}_{A} \oplus \mathrm{d}_{A}^{*}$ and a sum of linearized Cauchy-Riemann operators.

6.4. Equality of invariants. Suppose that every zero-area vortex is regular. In this case, every element of $\bar{M}_{n}^{K, \mathrm{fr}}\left(\mathbb{P}^{1}, X, d\right)_{[0, \rho]}$ is stable and regular for sufficiently small $\rho$, and so the projection $\bar{M}_{n}^{K, \mathrm{fr}}\left(\mathbb{P}^{1}, X, d\right)_{[0, \rho]} \rightarrow \bar{M}_{n}^{K}\left(\mathbb{P}^{1}, X, d\right)_{[0, \rho]}$ has the structure of an orbifold principal $K^{n}$-bundle. The evaluation maps extend over the cobordism and the boundary relation $\left[\bar{M}_{n}^{K,}\left(\mathbb{P}^{1}, X, d\right)_{0}\right]=\left[\bar{M}_{n}^{K}\left(\mathbb{P}^{1}, X, d\right)_{\rho}\right]$ in $H\left(\bar{M}_{n}^{K}\left(\mathbb{P}^{1}, X, d\right)_{[0, \rho]}, \mathbb{Q}\right)$ gives an equality of $\rho$ and 0 -vortex invariants.

\section{REFERENCES}

[1] K. Behrend. Gromov-Witten invariants in algebraic geometry. Invent. Math., 127(3):601-617, 1997.

[2] K. Behrend and B. Fantechi. The intrinsic normal cone. Invent. Math., 128(1):45-88, 1997.

[3] K. Behrend and Yu. Manin. Stacks of stable maps and Gromov-Witten invariants. Duke Math. J., 85(1):1-60, 1996.

[4] A. Bertram, I. Ciocan-Fontanine, and B. Kim. Gromov-Witten invariants for abelian and nonabelian quotients. J. Algebraic Geom., 17(2):275-294, 2008.

[5] K. Cieliebak, A. Rita Gaio, I. Mundet i Riera, and D. A. Salamon. The symplectic vortex equations and invariants of Hamiltonian group actions. J. Symplectic Geom., 1(3):543-645, 2002.

[6] K. Cieliebak, A. R. Gaio, and D. A. Salamon. J-holomorphic curves, moment maps, and invariants of Hamiltonian group actions. Internat. Math. Res. Notices, (16):831-882, 2000.

[7] T. Coates and A. Givental. Quantum Riemann-Roch, Lefschetz and Serre. Ann. of Math. (2), 165(1):15-53, 2007.

[8] S. K. Donaldson and P. Kronheimer. The geometry of four-manifolds. Oxford Mathematical Monographs. Oxford University Press, New York, 1990.

[9] W. Fulton and R. MacPherson. A compactification of configuration spaces. Ann. of Math. (2), 139(1):183-225, 1994.

[10] T. Graber and R. Pandharipande. Localization of virtual classes. Invent. Math., 135(2):487518, 1999.

[11] D. Halpern-Leistner. Remarks on Theta-stratifications and derived categories. arXiv: 1502.03083 .

[12] A. R. Pires Gaio and D. A. Salamon. Gromov-Witten invariants of symplectic quotients and adiabatic limits. J. Symplectic Geom., 3(1):55-159, 2005.

[13] A. B. Givental. Equivariant Gromov-Witten invariants. Internat. Math. Res. Notices, (13):613663, 1996.

[14] E. Gonzalez and C. Woodward. Area-dependence in gauged Gromov-Witten theory. arXiv:0811.3358

[15] E. Gonzalez and C. Woodward. Deformations of symplectic vortices. Ann. Global Anal. Geom., 39(1):45-82, 2011.

[16] A. Grothendieck. Cohomologie locale des faisceaux cohérents et théorèmes de Lefschetz locaux et globaux (SGA 2). Documents Mathématiques (Paris) [Mathematical Documents (Paris)], 4. Société Mathématique de France, Paris, 2005. Séminaire de Géométrie Algébrique du Bois Marie, 1962, Augmenté d'un exposé de Michèle Raynaud. [With an exposé by Michèle Raynaud], With a preface and edited by Yves Laszlo, Revised reprint of the 1968 French original.

[17] V. W. Guillemin and S. Sternberg. Supersymmetry and equivariant de Rham theory. SpringerVerlag, Berlin, 1999. With an appendix containing two reprints by Henri Cartan. 
[18] L. C. Jeffrey and F. C. Kirwan. Localization for nonabelian group actions. Topology, 34:291327, 1995.

[19] R. Joshua. Riemann-Roch for algebraic stacks. I. Compositio Math., 136(2):117-169, 2003.

[20] R. Joshua. Riemann-Roch for algebraic stacks:III virtual structure sheaves and virtual fundamental classes. Preprint.

[21] G. Kempf and L. Ness. The length of vectors in representation spaces. In K. Lønsted, editor, Algebraic Geometry, volume 732 of Lecture Notes in Mathematics, pages 233-244, Copenhagen, 1978, 1979. Springer-Verlag, Berlin-Heidelberg-New York.

[22] F. C. Kirwan. Cohomology of Quotients in Symplectic and Algebraic Geometry, volume 31 of Mathematical Notes. Princeton Univ. Press, Princeton, 1984.

[23] F. C. Kirwan. Partial desingularisations of quotients of nonsingular varieties and their Betti numbers. Ann. of Math. (2), 122:41-85, 1985.

[24] Y.-P. Lee. Quantum K-theory. I. Foundations. Duke Math. J., 121(3):389-424, 2004.

[25] J. Lurie. Derived Algebraic Geometry XII: Proper Morphisms, Completions, and the Grothendieck Existence Theorem. Preprint, November 2011.

[26] S. Martin. Symplectic quotients by a nonabelian group and by its maximal torus. math.SG/0001002.

[27] D. McDuff and D. Salamon. J-holomorphic curves and symplectic topology, volume 52 of American Mathematical Society Colloquium Publications. American Mathematical Society, Providence, RI, 2004.

[28] I. Mundet i Riera. A Hitchin-Kobayashi correspondence for Kähler fibrations. J. Reine Angew. Math., 528:41-80, 2000.

[29] I. Mundet i Riera. Hamiltonian Gromov-Witten invariants. Topology, 42(3):525-553, 2003.

[30] L. Ness. A stratification of the null cone via the moment map. Amer. J. Math., 106(6):12811329, 1984. with an appendix by D. Mumford.

[31] A. Ott. Removal of singularities and Gromov compactness for symplectic vortices, 2009. To appear in Jour. Symp. Geom. arXiv.org:0912.2500.

[32] A. Ramanathan. Moduli for principal bundles over algebraic curves. I. Proc. Indian Acad. Sci. Math. Sci., 106(3):301-328, 1996.

[33] M. Romagny. Group actions on stacks and applications. Michigan Math. J., 53(1):209-236, 2005.

[34] T. Schürg. Deriving Deligne-Mumford stacks with perfect obstruction theories. arXiv:1005.3945

[35] T. Schürg, B. Toën, G. Vezzosi. Derived algebraic geometry, determinants of perfect complexes, and applications to obstruction theories for maps and complexes. arXiv:1102.1150.

[36] C. Teleman. The quantization conjecture revisited. Ann. of Math. (2), 152(1):1-43, 2000.

[37] C. Teleman and C. T. Woodward. The index formula for the moduli of $G$-bundles on a curve. Ann. of Math. (2), 170(2):495-527, 2009.

[38] V. Tonita. A virtual Kawasaki formula. arXiv:1110.3916.

[39] J. Wehrheim. Vortex invariants and toric manifolds. arXiv:0812.0299.

[40] K. Wehrheim. Uhlenbeck compactness. EMS Series of Lectures in Mathematics. European Mathematical Society (EMS), Zürich, 2004.

[41] C. Woodward. Localization via the norm-square of the moment map and the two-dimensional Yang-Mills integral. J. Symp. Geom., 3(1):17-55, 2006.

[42] C. Woodward. Moment maps and geometric invariant theory. arXiv:0912.1132, volume 1 of Les cours du C.I.R.M., pages 55-98. 2010. 
Department of Mathematics University of Massachusetts Boston 100 William T. Morrissey Boulevard Boston, MA 02125

E-mail address: eduardo@math.umb.edu

Mathematics-Hill Center, Rutgers University, 110 Frelinghuysen Road, Piscataway, NJ 08854-8019, U.S.A.

E-mail address: ctw@math.rutgers.edu 